Pacific

Journal of

Mathematics

\title{
A FLAG STRUCTURE ON \\ A CUSPED HYPERBOLIC 3-MANIFOLD
}

ELISHa FALbEL AND RAFAel SANTOS THEBALdI 


\title{
A FLAG STRUCTURE ON A CUSPED HYPERBOLIC 3-MANIFOLD
}

\author{
Elisha FALBEL AND RAFAEL SANTOS ThEBALdi
}

\begin{abstract}
A flag structure on a 3-manifold is an $(X, G)$ structure where $G=\operatorname{SL}(3, \mathbb{R})$ and $X$ is the space of flags on the 2-dimensional projective space. We construct a flag structure on a cusped hyperbolic manifold with unipotent boundary holonomy. The holonomy representation can be obtained from a punctured torus group representation into $\operatorname{SL}(3, \mathbb{R})$ which is equivariant under a pseudo-Anosov.
\end{abstract}

\section{Introduction}

A flag structure on a 3-manifold is an $(X, G)$ structure, where $G=\operatorname{SL}(3, \mathbb{R})$ and $X$ is the space of flags on the 2-dimensional projective space, that is, the space of pairs: point and line containing it. The most direct construction of such structures starts with a real projective surface or orbifold. The projectivization of its tangent bundle is a Seifert manifold and has a natural flag structure. Other constructions on Seifert manifolds are studied in [Barbot 2001]. Note that projective structures on 3-manifolds concern instead the group SL(4, $\mathbb{R})$; see [Cooper et al. 2015].

Representations of fundamental groups of three manifolds into $\operatorname{SL}(3, \mathbb{R})$ were obtained in [Falbel et al. 2015], following the method described in [Bergeron et al. 2014]; see also the CURVE project [Falbel et al. 2015-]. A fundamental question is whether these representations correspond to holonomies of flag structures on the manifold.

The goal of this paper is to construct a flag structure on a cusped hyperbolic manifold with unipotent boundary holonomy; see Theorem 6.8. We introduce a general method of construction via gluings of tetrahedra which are defined on the flag space. The tetrahedra are canonical up to a finite choice related to an order on the 0 -skeleton of an ideal triangulation of the manifold, once one fixes a decoration (that is a choice of a flag at each vertex) satisfying certain compatibility conditions; see [Bergeron et al. 2014]. Definitions of simplices in Grassmannian spaces (although not containing the case of flag space) were also considered in [Gelfand and MacPherson 1982] and inspired our definition of tetrahedron. In

MSC2010: primary 57M50; secondary 57S30.

Keywords: flag structures, hyperbolic structures, SL(3, R) representations. 
the Appendix we describe a slicing of the tetrahedra which allows an algorithmic treatment of the many compatibility verifications needed in the paper.

The method presented here can be considered as a flag structure analog of Thurston's construction [1981] of hyperbolic structures on cusped manifolds by gluing ideal hyperbolic tetrahedra and of the construction of CR structures, as in [Falbel 2008].

The holonomy representation of the structure we obtained is not faithful. It turns out that the manifold m009 we analyzed here has holonomy group contained in a triangle group of type $(3,3,5)$; see the end of the Appendix. An isomorphic triangle group was obtained in [Deraux 2015], where the holonomy representation has values in $\mathrm{PU}(2,1)$. These representations are Galois conjugates, as explained in [Falbel et al. 2015]; indeed, they are all parametrized by solutions of a degree four irreducible polynomial in one variable. Two solutions correspond to conjugate representations in $\mathrm{PU}(2,1)$ and the other two to two dual flag structures.

It is interesting to remark that the manifold m009 is fibered over the circle with fiber a punctured torus. The representation into $\operatorname{SL}(3, \mathbb{R})$ of the fiber surface group is then equivariant with respect to the mapping class group element defining the bundle.

\section{Flag structures on 3-manifolds}

A flag structure on a 3-manifold is an $(X, G)$ structure, where $X$ is a homogeneous space described in the following paragraph and $G=\operatorname{SL}(3, \mathbb{R})=\operatorname{PGL}(3, \mathbb{R})$.

The homogeneous space $X$ is the space of flags in $\mathbb{P}\left(\mathbb{R}^{3}\right)$. An affine flag in $V=\mathbb{R}^{3}$ is a pair (line, plane), the line belonging to the plane. They project to flags in $\mathbb{P}(V)$, that is, pairs (point, line). Using the dual vector space $V^{*}$ and the projective spaces $\mathbb{P}(V)$ and $\mathbb{P}\left(V^{*}\right)$, define the spaces of flags $\mathscr{F} l$ by the following:

$$
\mathscr{F} l=\left\{([p],[l]) \in \mathbb{P}(V) \times \mathbb{P}\left(V^{*}\right) \mid l(p)=0\right\} .
$$

The action of $\operatorname{SL}(3, \mathbb{R})$ on $V$ induces an action on $\mathbb{P}(V) \times \mathbb{P}\left(V^{*}\right)$. Indeed, identify $V$ and $V^{*}$ using the canonical scalar product and then, via this identification, the contragredient action (that is $g \cdot v=\left(g^{-1}\right)^{T} v$ ) on $V^{*}$. We denote by $\pi_{1}$ and $\pi_{2}$ the two projections of $\mathscr{F} l$ into $\mathbb{P}(V)$ and $\mathbb{P}\left(V^{*}\right)$, respectively.

Observe that

$$
\mathscr{F} l=\mathrm{SL}(3, \mathbb{R}) / B,
$$

where $B$ is the Borel subgroup of upper triangular matrices in $\operatorname{SL}(3, \mathbb{R})$. The flag space is identified with the projectivization of the tangent bundle to $P(V)$, and the differential action of $\operatorname{SL}(3, \mathbb{R})$ on the tangent bundle induces the above action. Observe that, in fact, $\operatorname{SL}(3, \mathbb{R})$ acts on the unit tangent bundle of $P(V)$ (which has 
$S^{3}$ as a double cover); therefore, the double cover of $\operatorname{SL}(3, \mathbb{R}$ ) (which is simply connected) acts on the sphere $S^{3}$.

Definition 2.1. A flag structure on a 3-manifold $M$ is a $(\mathscr{F} l, \operatorname{SL}(3, \mathbb{R}))$-structure on that manifold.

The involution $\Theta(v, w)=(w, v)$ on $\mathscr{F} l$ and the Cartan involution $\theta(g)=\left(g^{-1}\right)^{T}$ on $\operatorname{SL}(3, \mathbb{R})$ satisfy

$$
\Theta \circ g=\theta(g) \circ \Theta .
$$

Given a flag structure on a 3-manifold, we call a dual flag structure the structure obtained by using transition functions composed with $\theta$.

2.2. Coordinates in $\mathbb{P}(\boldsymbol{V})$. To facilitate visualization of the flags we will choose a chart (called the preferred chart) on $\mathbb{P}(V)$. Consider the hyperplane in $\mathbb{R}^{3}$ defined by the three basis unit vectors, that is

$$
x+y+z=1 .
$$

The chart is defined by projecting lines passing through the origin in that hyperplane and imposing that

$$
[1,0,0] \mapsto(0,0), \quad[0,1,0] \mapsto(1,0), \quad[0,0,1] \mapsto(0,1)
$$

The chart is defined on the complement of the projectivization of the plane $x+y+z=0$ and has the expression

$$
[x, y, z] \mapsto\left(\frac{y}{x+y+z}, \frac{z}{x+y+z}\right) .
$$

Observe that, on the hyperplane, $[x, y, z] \mapsto(y, z)$ and $[1,1,1] \mapsto\left(\frac{1}{3}, \frac{1}{3}\right)$.

Given a flag $[[x, y, z],[a, b, c]]$, with $x+y+z=1$, the point $[x, y, z]$ and the line on $\mathbb{P}(V)$ defined by the image of the plane orthogonal to the vector $(a, b, c)$ are described in the chart above by:

- the point $(y, z)$,

- the line defined by the vector $(a-c, b-a)$ passing through the point $(y, z)$.

Therefore, the line makes an angle $\theta$ with the first coordinate axis satisfying

$$
\tan \theta=\frac{b-a}{a-c}
$$

with the horizontal direction. Figure 1 shows three flags corresponding to planes passing through the three basis vectors in $\mathbb{R}^{3}$. 


$$
\overrightarrow{p_{3}}
$$
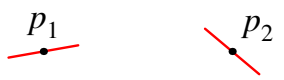

Figure 1. Three flags corresponding to planes passing through the three basis vectors in $\mathbb{R}^{3}$.

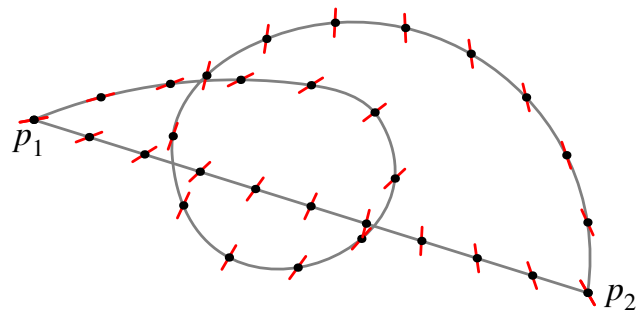

Figure 2. Two simple paths of flags projected into $\mathbb{P}(V)$.

\section{Edges}

One can join a pair of flags by simple paths (see Figure 2), but there is a canonical construction of a unique line containing two flags.

Consider two flags in generic position, that is, $f_{1}=\left(p_{1}, l_{1}\right), f_{2}=\left(p_{2}, l_{2}\right)$ such that $l_{i}\left(p_{j}\right) \neq 0$ if $i \neq j$. The action of $\operatorname{SL}(3, \mathbb{R})$ is transitive on these pairs. There exists a unique point $p_{12}$ such that $l_{i}\left(p_{12}\right)=0$, for $i=1,2$. Up to the action of $\mathrm{SL}(3, \mathbb{R})$ we can normalize so that

$$
p_{1}=(1,0,0), l_{1}=(0,1,0) \quad \text { and } \quad p_{2}=(0,1,0), l_{2}=(1,0,0) .
$$

The intersection point of the two lines is $p_{12}=(0,0,1)$. Projective transformations fixing the three points are diagonal and they preserve the line $\left[p_{1}, p_{2}\right]$. For each line $l$ passing through $p_{12}$ we consider its intersection $p$ with the line $\left[p_{1}, p_{2}\right]$; see Figure 3 . This defines a circle of flags $(p, l)$ containing $f_{1}$ and $f_{2}$. It is divided

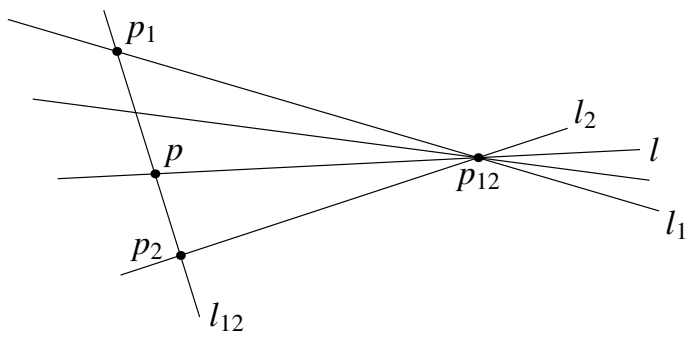

Figure 3. A segment between two flags. 


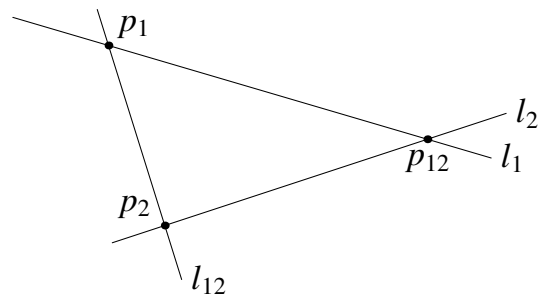

Figure 4. Points and lines fixed by $H_{12}^{0}$.

into two segments with two given flags as boundaries. Following [Gelfand and MacPherson 1982], we let $H_{12}^{0}$ be the connected component of the identity of the group preserving the points $p_{1}, p_{2}, p_{12}$. It preserves the lines $l_{1}, l_{2}, l_{12}$ (see Figure 4), and the two segments are orbits of its action on the space of flags whose closure contains the flags $f_{1}$ and $f_{2}$. In the normalization above we have

$$
H_{12}^{0}=\left[\begin{array}{ccc}
h_{1} & 0 & 0 \\
0 & h_{2} & 0 \\
0 & 0 & h_{3}
\end{array}\right]
$$

with $h_{i}>0$. The circle of flags is given by

$$
p=\left[\lambda_{1}, \lambda_{2}, 0\right], \quad l=\left[\lambda_{2},-\lambda_{1}, 0\right] .
$$

More generally, if $f_{1}=\left(p_{1}, l_{1}\right), f_{2}=\left(p_{2}, l_{2}\right)$ are two flags in generic position, then the line containing the flags is

$$
\left(\lambda_{1} p_{1}+\lambda_{2} p_{2}, \frac{\lambda_{2}}{l_{2}\left(p_{1}\right)} l_{2}-\frac{\lambda_{1}}{l_{1}\left(p_{2}\right)} l_{1}\right) .
$$

The line is divided into two segments, corresponding to the relative signs of $\lambda_{1}$ and $\lambda_{2}$. Observe that, if the two flags are in the preferred chart, then only one of the segments is contained in the chart. Indeed, one of the flags in the circle is not in the preferred chart as its corresponding point is at infinity for that chart.

The next lemma states a simple property of a segment between two flags. It is the basic technical result we need to construct the tetrahedra of flags and will be repeatedly used in the analysis of the example in the last section.

Lemma 3.1 (monotonicity lemma). Let $f_{1}=\left(p_{1}, l_{1}\right), f_{2}=\left(p_{2}, l_{2}\right)$ be two flags. Suppose they are contained in the preferred chart and the angles $\theta_{1}$ and $\theta_{2}$ of the lines in the chart coordinates satisfy $\theta_{1} \leq \theta_{2}$. Then, along the finite segment from $f_{1}$ to $f_{2}$, the angles of the projected lines are increasing (and satisfy $\theta_{1} \leq \theta \leq \theta_{2}$ ).

If $f_{2}^{\prime}=\left(p_{2}, l_{2}^{\prime}\right)$ is another flag such that $\theta_{2}<\theta_{2}^{\prime}$ then, along the corresponding segment from $f_{1}$ to $f_{2}^{\prime}$, the angles of the projected lines satisfy $\theta<\theta^{\prime}$. 
Proof. We use the preferred chart (see Figure 3). The two lines $l_{1}$ and $l_{2}$ intersect at a point $p_{12}$ (which might be at infinity). The lines belonging to the finite segment of flags between $f_{1}$ and $f_{2}$ are lines passing through $p$ between $l_{1}$ and $l_{2}$. The angle of each line is also read at $p_{12}$ and is clearly a monotone function between $l_{1}$ and $l_{2}$.

For the second assertion, in the preferred chart, suppose that the vertices $p_{1}, p_{2}$, and $p_{12}$ determine a finite triangle (otherwise the lines $l_{1}$ and $l_{2}$ are parallel and the analysis is simpler). If $f_{2}^{\prime}=\left(p_{2}, l_{2}^{\prime}\right)$ is another flag such that $\theta_{2} \leq \theta_{2}^{\prime}$, then $p_{12}^{\prime}$, the intersection of $l_{2}^{\prime}$ and $l_{1}$, is on the side $\left[p_{1}, p_{12}\right]$. Therefore, the line passing through $p_{12}$ and a point $t$ in $\left[p_{1}, p_{2}\right]$ has smaller angle than the line passing through $p_{12}^{\prime}$ and $t$.

\section{Triangles}

By a generic configuration of flags $\left(\left[p_{i}\right],\left[l_{i}\right]\right), 1 \leq i \leq n+1$, we mean $n+1$ points $\left[p_{i}\right]$ in general position and $n+1$ lines $l_{i}$ in $\mathbb{P}(V)$ such that $l_{j}\left(p_{i}\right) \neq 0$ if $i \neq j$. Recall that a configuration of ordered points in $\mathbb{P}(V)$ is said to be in general position when no three points are contained in the same line. Notice that we give priority to the points in the above definition and don't impose that the lines are in generic position.

Let $\left(e_{1}, e_{2}, e_{3}\right)$ be the canonical basis of $V$ and $\left(e_{1}^{*}, e_{2}^{*}, e_{3}^{*}\right)$ its dual basis. Up to the action of $\operatorname{SL}(3, \mathbb{R})$, a generic configuration of three flags $\left(\left[p_{i}\right],\left[l_{i}\right]\right), 1 \leq i \leq 3$, can be normalized in these coordinates as

- $p_{1}=(1,0,0), \quad l_{1}=(0,1,1)$,

- $p_{2}=(0,1,0), \quad l_{2}=(1,0,1)$, and

- $p_{3}=(0,0,1), \quad l_{3}=(z, 1,0)$, with $z \neq 0$.

Therefore, the only invariant of a generic configuration of three flags, up to $\operatorname{SL}(3, \mathbb{R})$, is the triple ratio, given by

$$
z=\frac{l_{1}\left(p_{2}\right) l_{2}\left(p_{3}\right) l_{3}\left(p_{1}\right)}{l_{1}\left(p_{3}\right) l_{2}\left(p_{1}\right) l_{3}\left(p_{2}\right)} \in \mathbb{R}^{\times}
$$

Observe that the three lines of the triple of flags are linearly independent if and only if $z \neq-1$.

Given three flags in general position, $f_{1}=\left(p_{1}, l_{1}\right), f_{2}=\left(p_{2}, l_{2}\right), f_{3}=\left(p_{3}, l_{3}\right)$, we may form a triangle (a 1-skeleton as in Figure 5) by choosing three edges as above. There are 8 possible choices, namely for each pair of flags in a chart one can choose either the bounded segment or the unbounded segment with end points given by the two flags.

Fixing a choice of edges, we define a face as an embedded 2-simplex whose boundary is the union of the three edges. Observe that this imposes a restriction on the 1-simplex; it should be null-homotopic. This is equivalent to the condition that 


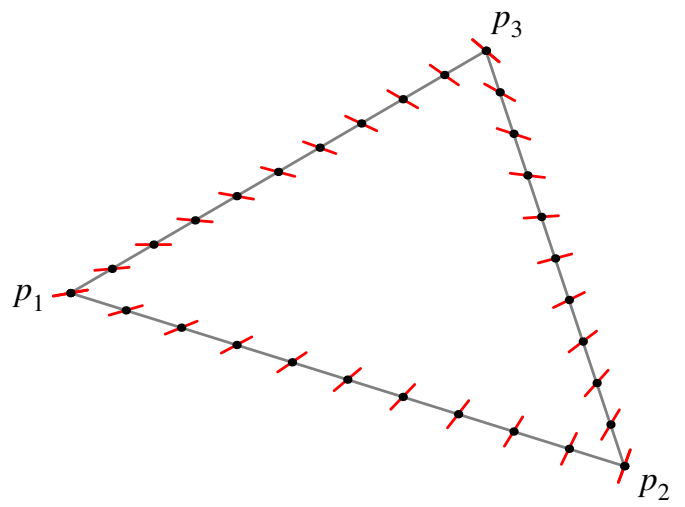

Figure 5. A triangle of flags projected into $\mathbb{P}(V)$.

both projections by $\pi_{1}$ and $\pi_{2}$ of the 1 -skeleton should be null-homotopic. If the edges are as in the previous section, then there is a restriction on the triple ratio of a triple of flags:

Lemma 4.1. A triple of flags defines a null-homotopic canonical 1-skeleton if and only if the triple ratio of the three flags is negative. In that case there are precisely four canonical 1-skeletons which are null-homotopic.

The proof of the lemma consists of comparing the two possible situations in Figure 6, corresponding to negative and positive triple ratios respectively. To obtain the sign of the triple ratio one simply counts the number of times the lines separate the points not contained in them.
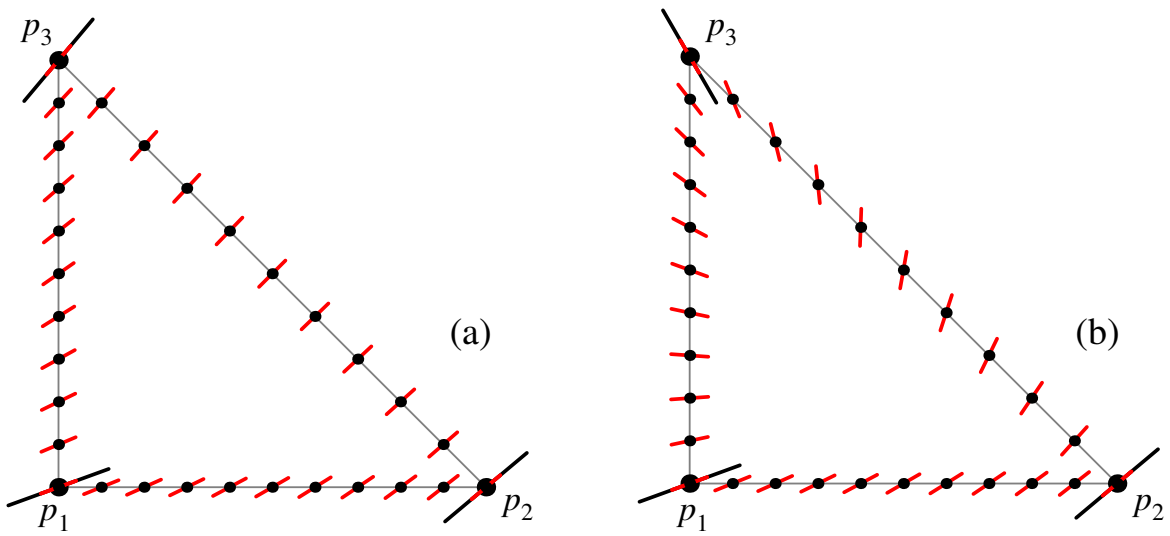

Figure 6. Each diagram shows three flags and the segments joining them, projected in the preferred chart. We only draw the finite triangle. The Euler number of a vector field parallel to the line field along the triangle is 0 in case (a) and has absolute value 1 in case (b). The triple ratio is negative in (a) and positive in (b). 


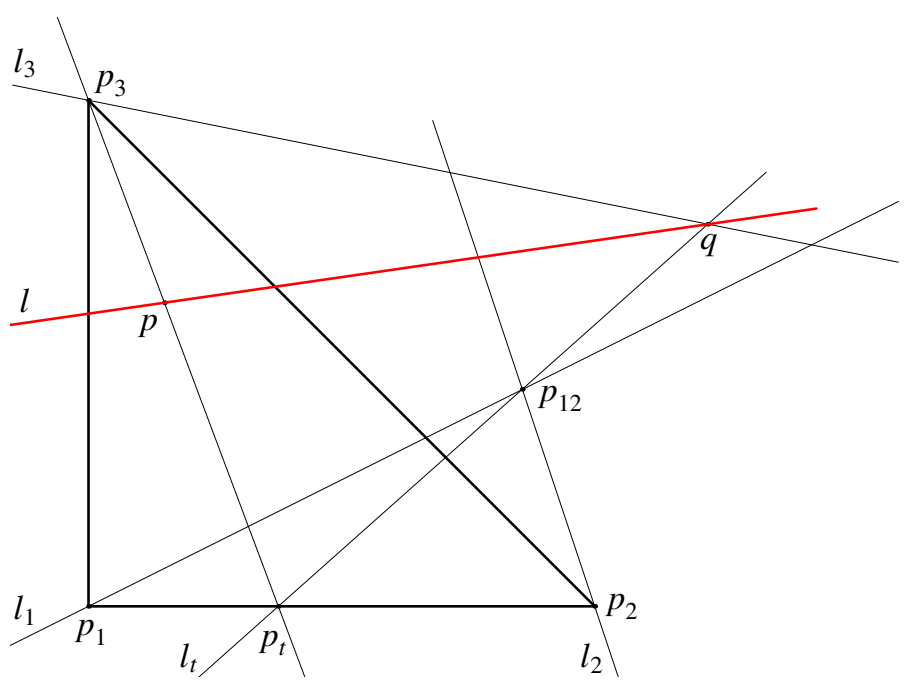

Figure 7. A synthetic construction of the flag $(p, l)$ in the face $F_{312}$. The triple ratio is negative and $f_{3}$ is the source of the face.

Once the 1-skeleton is defined, we should define a 2-simplex whose boundary is the given 1-skeleton. A particular canonical choice is given as a union of segments:

Definition 4.2. A face $F_{123}$ in the flag space, with vertices $f_{i}, i=1,2,3$ (with negative triple ratio) and a choice of edges $\left[f_{1}, f_{2}\right],\left[f_{2}, f_{3}\right],\left[f_{3}, f_{1}\right]$, is the 2-skeleton, which is the union of segments between $f_{1}$ and $f_{t}$, where $f_{t} \in\left[f_{2}, f_{3}\right]$; that is,

$$
F_{123}=\left\{f \in \mathscr{F} l \mid f \in\left[f_{1}, f_{t}\right] \text { for } f_{t} \in\left[f_{2}, f_{3}\right]\right\} .
$$

The flag $f_{1}$ is called the source of the face. For example, in Figure 7 the edges are bounded segments, $f_{3}$ is the source of the face $F_{312}$ and the triple ratio is negative. Notice that with the same vertices and edges we can construct the face $F_{213}$ in the same way, but we can't obtain the face $F_{123}$ because as $f_{t} \in\left[f_{2}, f_{3}\right]$, there is a flag $f_{0}=\left(p_{0}, l_{0}\right) \in\left[f_{2}, f_{3}\right]$ such that $p_{1} \in l_{0}$, so the flags $f_{0}$ and $f_{1}$ are not in general position. Thus, given a triple of flags with negative triple ratio, the surface obtained is embedded with boundary the union of edges only for two good choices of the source.

If the triple of flags has positive triple ratio it will be impossible to fill up a triangle unless we change the 1-skeleton in the following way: in the configuration represented in Figure 8, there is a flag $f_{0}=\left(p_{0}, l_{0}\right) \in\left[f_{2}, f_{3}\right]$ such that $p_{1} \in l_{0}$, so the flags $f_{0}$ and $f_{1}$ are not in general position. In order to define the triangle we should add, along the points $p \in\left[p_{0}, p_{1}\right)$ the flags $\left(p, l_{0}\right)$ and over the point $p_{1}$ the flags $\pi_{1}^{-1}\left(p_{1}\right)$. In this way the projection of the 1 -skeleton is twice the generator 


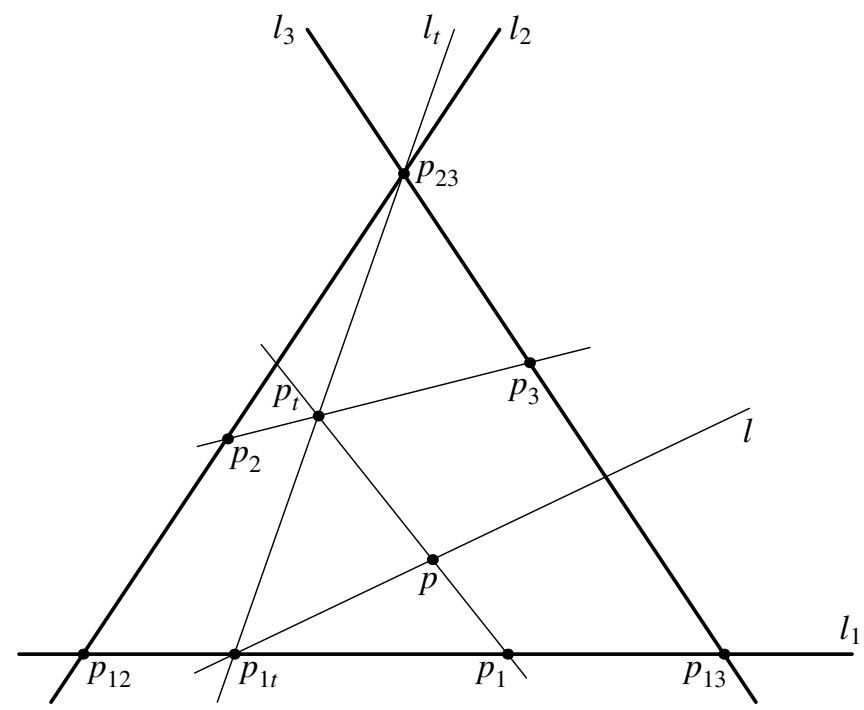

Figure 8. A synthetic construction of the flag $(p, l)$ in the face $F_{123}$. The triple ratio is positive.

and therefore it is null-homotopic. In this paper, though, we will only use triples with negative ratio and good choices of the source of the face.

The 2-skeleton determines a triangle $T_{123} \subset \mathbb{P}(V)$ when projected by $\pi_{1}$ and $T_{123}^{*} \subset \mathbb{P}\left(V^{*}\right)$ when projected by $\pi_{2}$. That is,

$$
\pi_{1}\left(F_{123}\right)=T_{123}, \quad \pi_{2}\left(F_{123}\right)=T_{123}^{*} .
$$

\section{Coordinates on a flag tetrahedron}

In this section we recall the coordinates parametrizing configurations of four flags in the projective space $\mathbb{P}\left(\mathbb{R}^{3}\right)$, as in [Bergeron et al. 2014; Falbel et al. 2015]; also see [Fock and Goncharov 2007; Garoufalidis et al. 2011].

5.1. Coordinates for a tetrahedron offlags. Let $\left(\left[p_{i}\right],\left[l_{i}\right]\right), 1 \leq i \leq 4$, be a generic tetrahedron. Arrange these flags symbolically on a tetrahedron 1234 as in Figure 9. We define a set of 12 coordinates on the edges of the tetrahedron, one for each oriented edge.

To define the coordinate $z_{i j}$ associated to the edge $i j$, we first define $k$ and $l$ such that the permutation $(1,2,3,4) \mapsto(i, j, k, l)$ is even. The pencil of (projective) lines through the point $p_{i}$ is a projective line $\mathbb{P}_{1}(k)$. We have four points on this projective line: the line $\operatorname{ker}\left(l_{i}\right)$ and the each of the lines going through $p_{i}$ and one of the $p_{l}$, for $l \neq i$. We define $z_{i j}$ as the cross-ratio of four flags by

$$
z_{i j}:=\left[\operatorname{ker}\left(l_{i}\right),\left(p_{i} p_{j}\right),\left(p_{i} p_{k}\right),\left(p_{i} p_{l}\right)\right] .
$$




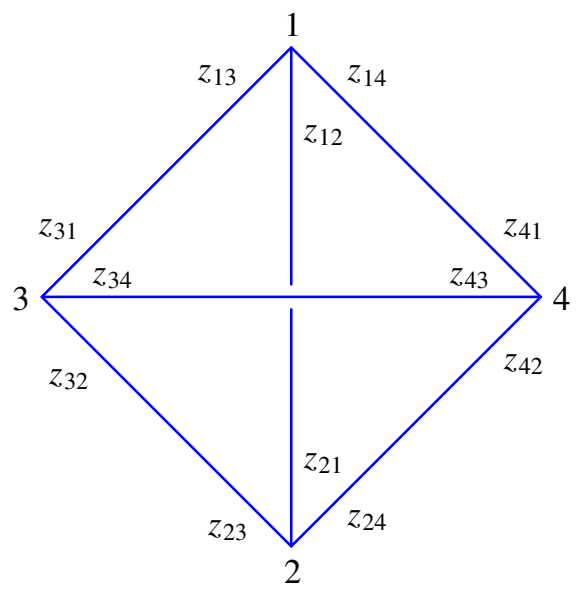

Figure 9. The $z$-coordinates.

We follow the usual convention that the cross-ratio of four points $p_{1}, p_{2}, p_{3}, p_{4}$ on a line is the value at $p_{4}$ of a projective coordinate taking value $\infty$ at $p_{1}, 0$ at $p_{2}$, and 1 at $p_{3}$. Figure 9 displays the coordinates.

At each face $(i j k)$, oriented as the boundary of the tetrahedron (1234), we associate the triple ratio:

$$
z_{i j k}=\frac{l_{i}\left(p_{j}\right) l_{j}\left(p_{k}\right) l_{k}\left(p_{i}\right)}{l_{i}\left(p_{k}\right) l_{j}\left(p_{i}\right) l_{k}\left(p_{j}\right)} .
$$

Observe that if the same face with opposite orientation $(i k j)$ is common to a second tetrahedron, then

$$
z_{i k j}=\frac{1}{z_{i j k}} .
$$

Of course there are relations between the whole set of coordinates. Fix an even permutation $(i, j, k, l)$ of $(1,2,3,4)$. First, for each face $(i j k)$, the triple ratio is the opposite of the product of all cross-ratios "leaving" this face:

$$
z_{i j k}=-z_{i l} z_{j l} z_{k l} .
$$

Second, the three cross-ratios leaving a vertex are algebraically related. For instance, in the vertex 1 ,

$$
z_{13}=\frac{1}{1-z_{12}} \quad \text { and } \quad z_{14}=1-\frac{1}{z_{12}},
$$

and analogously for the other vertices. The next proposition shows that a tetrahedron is uniquely determined, up to the action of $\operatorname{SL}(3, \mathbb{R})$, by four numbers.

Proposition 5.2 [Bergeron et al. 2014, Proposition 2.4.1]. The space of generic tetrahedra is parametrized by the 4-tuple $\left(z_{12}, z_{21}, z_{34}, z_{43}\right)$ of elements in $\mathbb{R} \backslash\{0,1\}$. 
In particular, one can normalize the coordinates of four flags up to the action of $\mathrm{SL}(3, \mathbb{R})$ as

$$
\begin{array}{ll}
f_{1}: & p_{1}=(1,0,0), \quad l_{1}=\left(0, z_{14},-1\right)=\left(-1,1-\frac{1}{z_{12}},-1\right), \\
f_{2}: & p_{2}=(0,1,0), \quad l_{2}=\left(\frac{1}{z_{24}}, 0,-1\right)=\left(1-z_{21}, 0,-1\right), \\
f_{3}: & p_{3}=(0,0,1), \quad l_{3}=\left(z_{34},-1,0\right), \\
f_{4}: & p_{4}=(1,1,1), \quad l_{4}=\left(z_{42}, \frac{1}{z_{41}},-1\right)=\left(\frac{1}{1-z_{43}}, \frac{z_{43}}{z_{43}-1},-1\right) .
\end{array}
$$

\section{Example: m009}

The manifold m009 is an open manifold which has a complete hyperbolic structure with finite volume. It is obtained by gluing three tetrahedra $T_{0}\left(u_{i j}\right), T_{1}\left(v_{i j}\right)$, and $T_{2}\left(w_{i j}\right)$ as shown in Figure 10.

The face identifications are

$$
\begin{aligned}
(234)^{0} \leftrightarrow(243)^{1}, & (142)^{0} \leftrightarrow(314)^{1}, & (134)^{0} \leftrightarrow(143)^{2}, \\
(123)^{0} \leftrightarrow(213)^{2}, & (142)^{1} \leftrightarrow(241)^{2}, & (123)^{1} \leftrightarrow(342)^{2} .
\end{aligned}
$$

In [Falbel et al. 2015], we obtained a particular realization of these tetrahedra by 4-tuples of flags giving rise to representations into $\operatorname{SL}(3, \mathbb{R})$ with unipotent boundary holonomy. The invariants of the 4-tuple of flags all depend on

$$
\gamma=-\frac{1}{2}+\frac{1}{2} \sqrt{5+4 \sqrt{5}}
$$
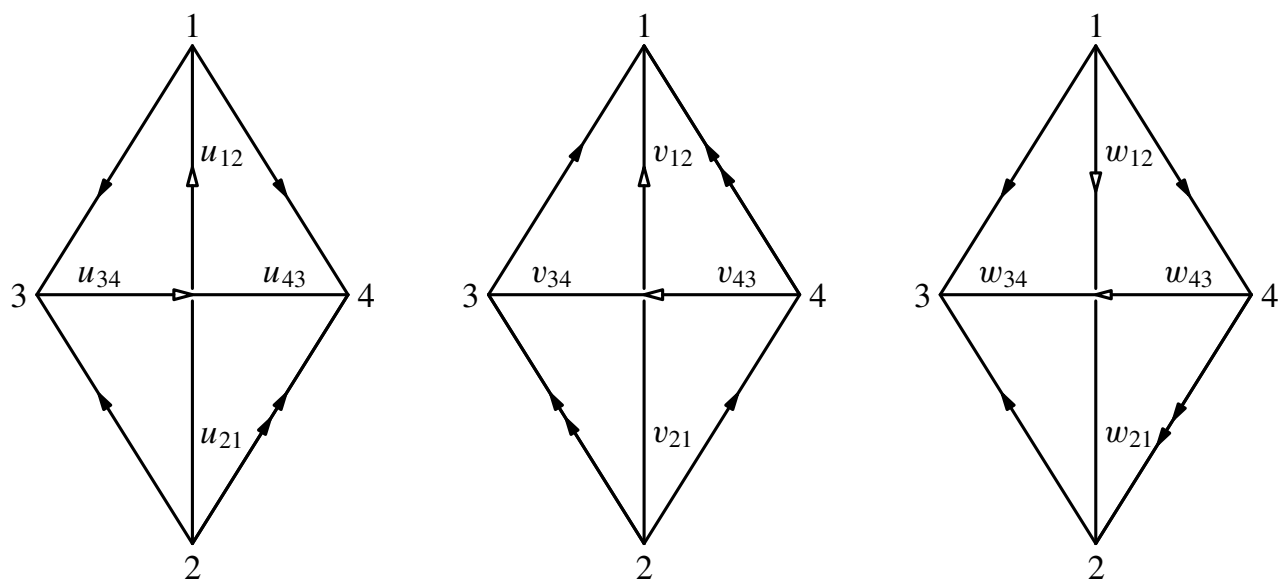

Figure 10. Three tetrahedra glued to obtain the manifold m009. The tetrahedra are numbered from 0 to 2 from left to right. 
Explicitly:

$$
\begin{array}{lll}
u_{12}=w_{34}=\frac{\gamma+3}{\gamma+1}, & u_{21}=w_{43}=\gamma, & u_{34}=w_{12}=\frac{\gamma-2}{\gamma}, \\
u_{43}=w_{21}=-1-\gamma, & v_{12}=v_{34}=\frac{1}{\gamma+3}, & v_{21}=v_{43}=\frac{1}{2-\gamma} .
\end{array}
$$

The group obtained has rank one boundary holonomy, and one can chose generators, called meridian $g_{M}$ and longitude $g_{L}$, satisfying $g_{M} g_{L}^{2}=1$.

The realization described above comes paired with another one giving rise to a dual flag structure. It is also related to a representation of the fundamental group in $\mathrm{PU}(2,1)$ with boundary holonomy of rank one, which seems to give rise to a uniformizable CR structure on m009 [Deraux 2015].

6.1. The tetrahedron $\boldsymbol{T}_{\mathbf{0}}$. Using the coordinates above, the four flags $f_{i}=\left[p_{i}, l_{i}\right]$, $1 \leq i \leq 4$, defining $T_{0}$ can be represented in the preferred chart, as in Figure 11 . Setting

$$
\kappa=2 \gamma+1=\sqrt{5+4 \sqrt{5}},
$$

the coordinates of the flags are

$$
\begin{aligned}
& f_{1}=\left[[1,0,0],\left[0, \frac{4}{5+\kappa},-1\right]\right] \approx[[1,0,0],[0,0.458,-1]], \\
& f_{2}=\left[[0,1,0],\left[\frac{3-\kappa}{2}, 0,-1\right]\right] \approx[[0,1,0],[-0.367,0,-1]],
\end{aligned}
$$

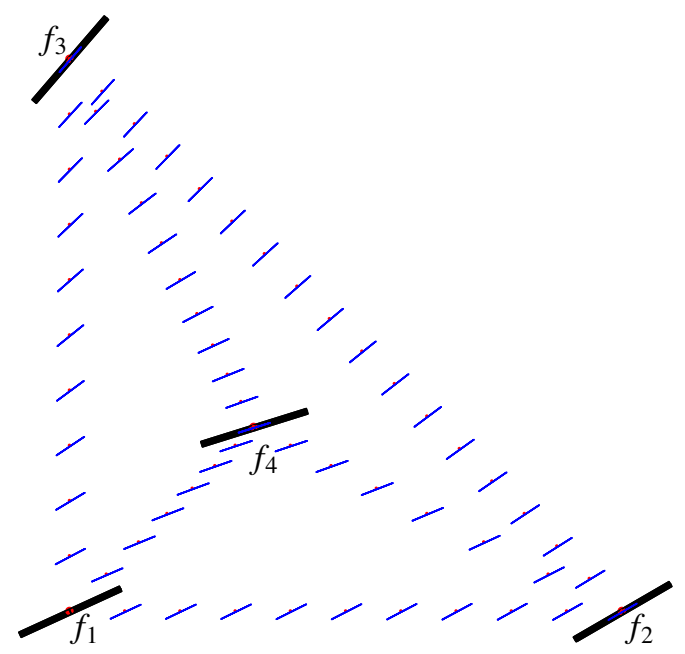

Figure 11. The four flags of tetrahedron $T_{0}$ and segments joining them projected in the preferred chart. Here $\theta_{4}<\theta_{1}<\theta_{2}<\theta_{3}$. 


$$
\begin{aligned}
& f_{3}=\left[[0,0,1],\left[\frac{-5+\kappa}{-1+\kappa},-1,0\right]\right] \approx[[0,0,1],[-0.463,-1,0]], \\
& f_{4}=\left[[1,1,1],\left[\frac{2}{3+\kappa}, \frac{1+\kappa}{3+\kappa},-1\right]\right] \approx[[1,1,1],[0.297,0.703,-1]] .
\end{aligned}
$$

The angles at each flag in the preferred chart are computed using formula (2.2.1):

$$
\begin{aligned}
& \tan \theta_{1}=\frac{4}{5+\kappa} \quad \Rightarrow \quad \theta_{1} \approx 0.43, \\
& \tan \theta_{2}=-\frac{-3+\kappa}{-5+\kappa} \quad \Longrightarrow \quad \theta_{2} \approx 0.53, \\
& \tan \theta_{3}=-\frac{-3+\kappa}{-5+\kappa} \quad \Longrightarrow \quad \theta_{3} \approx 0.86, \\
& \tan \theta_{4}=\frac{-1+\kappa}{5+\kappa} \quad \Longrightarrow \quad \theta_{4} \approx 0.30 .
\end{aligned}
$$

We choose the segments between the flags so that all of them are finite and contained in the preferred chart.

Proposition 6.2. The four flags defining $T_{0}$ and the 1-skeleton $E_{i j}$ (defined by the finite segments joining the flags $i$ and $j$ in the preferred chart) can be extended to $a$ simplex with faces $F_{314}^{0}, F_{342}^{0}, F_{412}^{0}, F_{312}^{0}$.

Proof. We first define an embedded 2-skeleton. In the last paragraph of the proof below we fill it up to a 3-simplex.

We need to construct the four faces of the tetrahedron and verify that their intersections are precisely their common edges. They are (writing $F_{i j k}^{0}=F_{i j k}$, etc., to simplify the notation):

$$
F_{314}, \quad F_{342}, \quad F_{412}, \quad F_{312} \text {. }
$$

Clearly, the first three faces only intersect in their common edges. The only verification to be done is on the intersection of these faces with $F_{312}$. We need to prove that

$$
F_{412} \cap F_{312}=E_{12}, \quad F_{314} \cap F_{312}=E_{31}, \quad F_{342} \cap F_{312}=E_{32} .
$$

The argument uses Lemma 3.1 in a simple way. We choose the preferred chart. Observe first, because $\theta_{4}<\theta_{1}<\theta_{2}$, that the segment $E_{12}$ has all flags with angles greater than the flags at the edges $E_{14}$. By the lemma, $F_{314} \cap F_{312}=E_{31}$.

Observe that the line from $p_{3}$ to $p_{4}$ intersects the edge $E_{12}$ at a point, say $p$, whose flag has angle $\theta>\theta_{4}$. Moreover, a simple drawing (see Figure 12) or computation shows that the intersection point of $l_{4}$ with the line $l_{2}$ is between $p_{2}$ and the intersection point between $l_{1}$ and $l_{2}$. This is sufficient to prove that the angle of a flag along the segment $E_{24}$ is smaller than the corresponding flag (along 


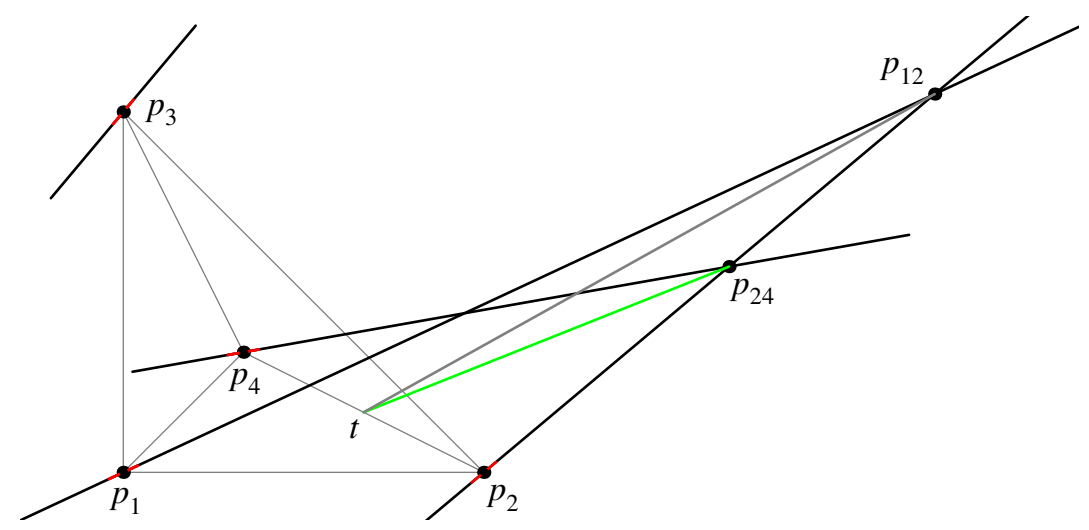

Figure 12. Comparison of two flags over a point $t \in p_{2} p_{4}$. At the point $t$ the flag of the face $F_{312}$ has greater angle than the one of the face $F_{342}$.

the segment whose projection contains $p_{3}$ and the projection of the flag in $E_{24}$ ) passing at the edge $E_{12}$. This implies, again by the lemma, that $F_{342} \cap F_{312}=E_{32}$.

To analyze $F_{412} \cap F_{312}$, observe that if $x$ belongs to the triangle $p_{1} p_{2} p_{4}$ and is to the left of the line $p_{3} p_{4}$, then, because $\theta_{1}<\theta_{2}$, the angle at $x$ along the line from $p_{3}$ is greater than the angle along the line from $p_{4}$. For a point to the right of the line $p_{3} p_{4}$, we conclude with an argument analogous to the previous paragraph. This implies again that $F_{412} \cap F_{312}=E_{12}$.

The last part of the proof consists in completing the 2-skeleton to a 3-simplex. We do it explicitly in the following way. For each point $p$ in the preferred coordinate chart in the triangle $p_{1} p_{2} p_{3}$, there are two angles $\theta \geq \theta^{\prime}$, the first one corresponding to the face $F_{312}$ and the other to one of the other three faces. We define a segment of flags for each of these points by varying the angle from the first angle (at face $\left.F_{312}\right)$ to the one on the other face. That is, we consider all flags passing through $p$ with angles $\phi$, with $\theta \geq \phi \geq \theta^{\prime}$, where we have strict inequality outside the edges of the triangle $p_{1} p_{2} p_{3}$.

6.3. The tetrahedra $\boldsymbol{T}_{\mathbf{1}}$ and $\boldsymbol{T}_{2}$. In Figure 13 we show the three tetrahedra glued according to $g_{1}:(243)^{1} \rightarrow(234)^{0}$ and $g_{2}:(142)^{2} \rightarrow(241)^{1}$. The three tetrahedra are $T_{0}, g_{1}\left(T_{1}\right)$, and $g_{1} g_{2}\left(T_{2}\right)$.

The points in the figure are projections of the flags

$$
f_{5}=\left[p_{5}, l_{5}\right]=g_{1}\left[p_{1}, l_{1}\right] \quad \text { and } \quad f_{6}=\left[p_{6}, l_{6}\right]=g_{1} g_{2}\left[p_{3}, l_{3}\right] .
$$

Due to the face pairings, the faces of $T_{1}$ and $T_{2}$ are in part determined by the choice of the faces of $T_{0}$, namely, for $T_{1}, F_{432}^{1}$, and $F_{134}^{1}$ and for $T_{2}, F_{413}^{2}$, and $F_{312}^{2}$ are determined. The remaining two pairs of faces can be chosen arbitrarily. 
Observe that $F_{432}^{1}$ and $F_{134}^{1}$ are represented, in the glued configuration, by $F_{342}$ and $F_{543}$, respectively. Also, $F_{413}^{2}$ and $F_{312}^{2}$ are represented by $F_{326}$ and $F_{625}$.

The definition of each filled tetrahedron follows the same line as for $T_{0}$, namely, in the preferred chart we fix a point which is a projection of two flags in two different faces. These two flags determine two angles. We then obtain a segment of flags defined by varying the angles between these two angles. The 3 -simplex is the union of those segments.

We have to verify compatibilities in the definition, namely, that the side pairings map the edges between themselves and that the tetrahedra defined by the faces above do not intersect except in their common faces. We state the compatibility of the edges as a lemma whose proof is a straightforward computation.

Lemma 6.4. The finite edges between the flags are compatible with the side pairings. That is, the face pairings map finite edges onto finite edges.

Proof. The compatibility of the vertices is already verified by the definition of the tetrahedra in the computations in [Falbel et al. 2015]. We need to verify that in Figure 13, finite segments are mapped to finite segments by the side pairings. The side pairings are given by the four maps $s_{3}, s_{4}, s_{5}, s_{6}$; see the Appendix. Clearly, each transformation $g_{a}:(i j k)^{m} \rightarrow\left(i^{\prime} j^{\prime} k^{\prime}\right)^{n}$ is completely determined by the vertices of the two faces. The polyhedron side pairings $s_{i}$ are determined by the $g_{a}$.

In our case, a tedious verification shows that all maps $s_{3}, s_{4}, s_{5}, s_{6}$ are such that they always map finite segments that are edges of a face of one tetrahedron of the

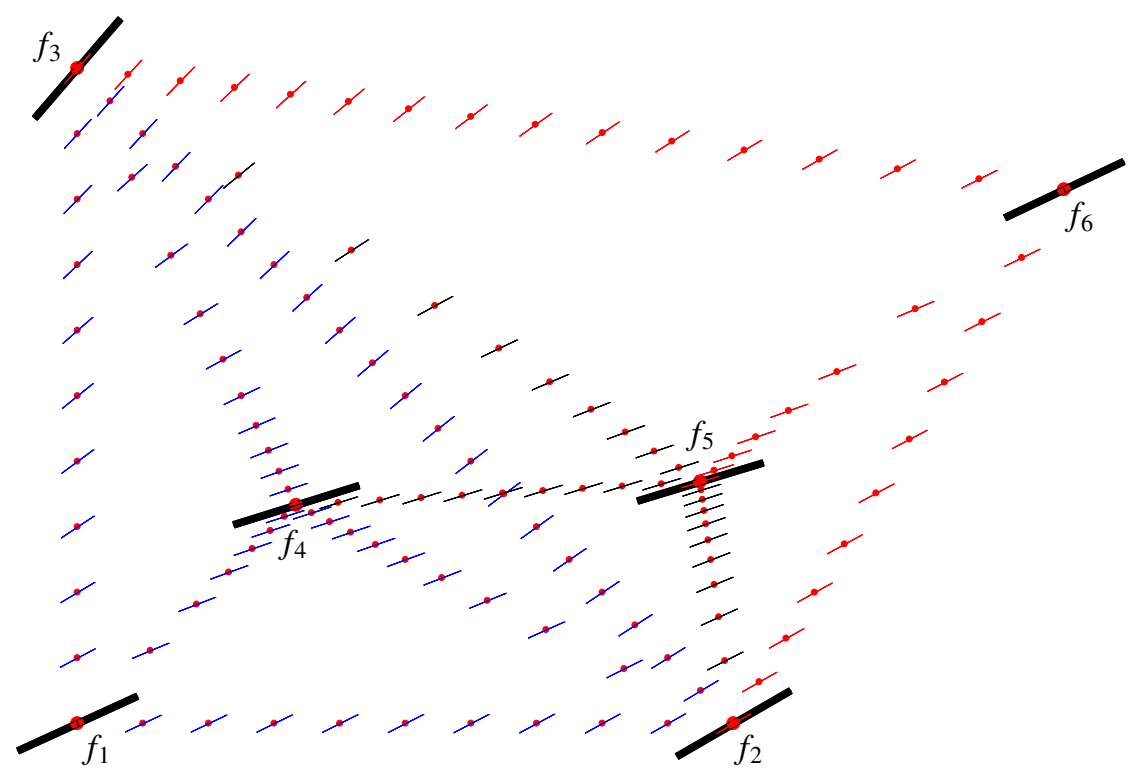

Figure 13. Gluing the 3 tetrahedra projected in the preferred chart. 
polyhedron into finite segments that are edges of the corresponding face in another tetrahedron of the polyhedron.

The next verification proves that $T_{0}, g_{1}\left(T_{1}\right)$, and $g_{1} g_{2}\left(T_{2}\right)$ are well defined and form a polyhedron in the flag space. That is, as for $T_{0}$, their faces intersect only at common edges. Finally, we prove that the three tetrahedra intersect only at common faces. The proof is a sequence of tedious arguments (Proposition A7), as in the proof that $T_{0}$ was well defined, but one can be convinced by carefully looking at Figure 13.

Proposition 6.5. The gluing of the three tetrahedra $T_{0}, g_{1}\left(T_{1}\right)$, and $g_{1} g_{2}\left(T_{2}\right)$ forms a polyhedron in the flag space.

6.6. The structure around the edges. There are three edges in the quotient manifold, represented by the edges $E_{23}, E_{24}$, and $E_{34}$ in the first tetrahedron $T_{0}$. As far as the topological gluing is concerned, the number of tetrahedra around each edge are 8, 4, and 6 respectively (we show the schematic diagram of the gluing for each edge in Figure 14). To prove that we have a genuine flag structure on the quotient manifold, we should prove that the gluing of the tetrahedra around each of the three edges has no branching. That is, that the gluing around each edge gives a neighborhood of the edge.

We state the result in the following proposition. Its proof is a tedious verification and is given in one particular case; see Proposition A9. We use a slicing of the tetrahedra to describe the behavior of the structures around the edges. Heuristically, one can understand the neighborhood of an edge by following the vertices of the tetrahedra that one adjoins to the edge. Turning around the edge corresponds to turning the angle of the projected line of the flag in the vertex in such a way that increasing the angle makes the tetrahedron go up and decreasing the angle makes the tetrahedron go down.

In Figure 15, we show that the 4 tetrahedra around the edge $E_{24}$ (the complete proof in this case is given in the Appendix). One can observe that the last point adjoined has the projected line of angle lower than the others. The tetrahedra adjoined will be below the original two. In Figure 16, we show 5 of the 6 tetrahedra around the edge $E_{34}$. Here we have to add three more points to the original 3 tetrahedra. Observe that the first two have lines of decreasing angle, but the last point increases the angle in order to complete the turn. In Figure 17, we show the vertices of the 8 tetrahedra around the edge $E_{23}$.

A detailed proof of the following proposition is given in Proposition A9 for the case $E_{34}$. For the others two gluings around $E_{23}$ and $E_{34}$, the proof follows the same lines.

Proposition 6.7. Along each of the three edges $E_{23}, E_{24}$, and $E_{34}$ the gluing of the tetrahedra defines a neighborhood. 


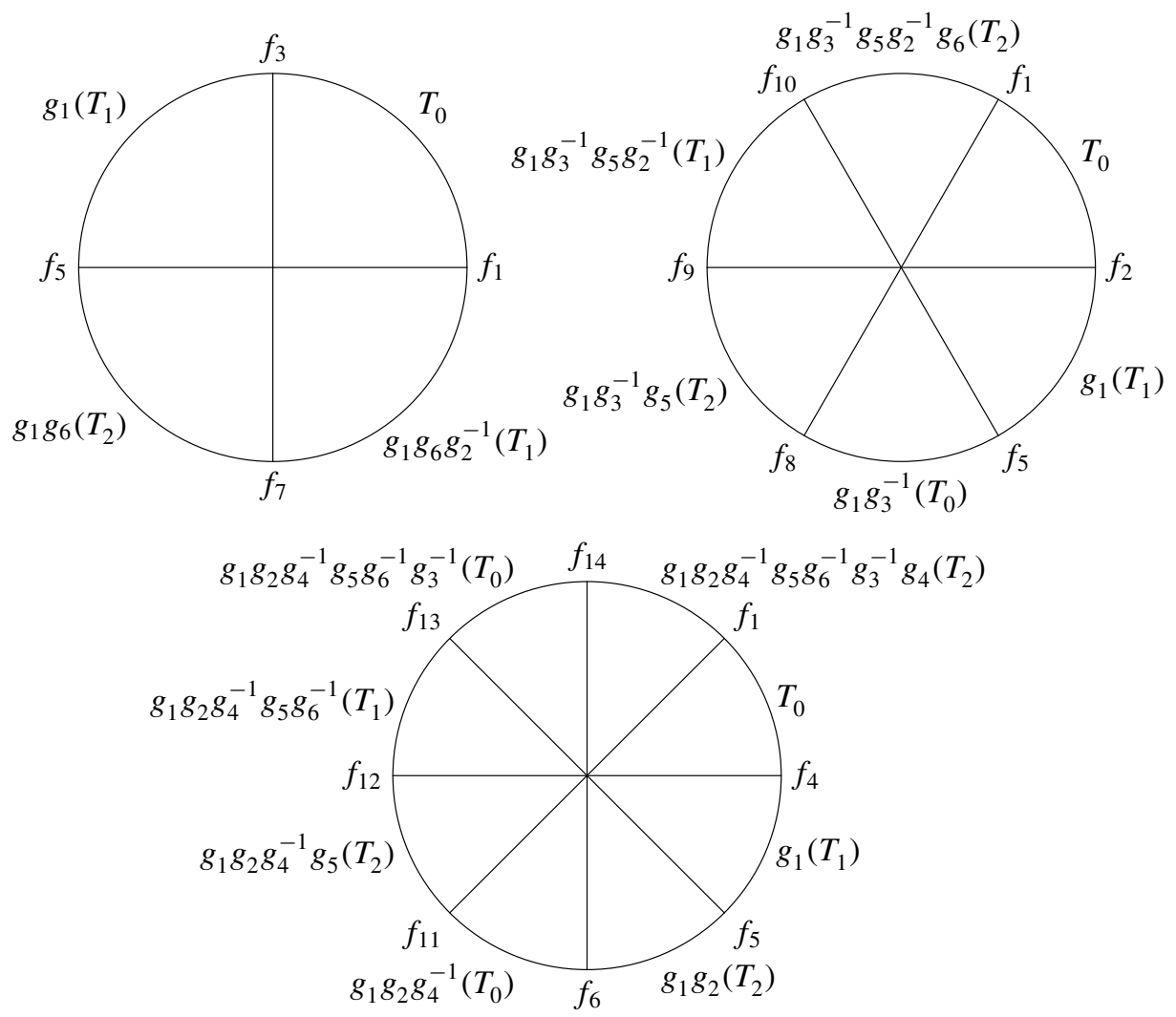

Figure 14. Top left: schematics of a neighborhood around the edge $E_{24}=\left[f_{2}, f_{4}\right]$, where the segments stand for the faces with the common edge $E_{24}$ denoted by the origin and the arcs and the regions between two segments stand for the neighborhoods contained in one tetrahedron. The remaining diagrams represent a neighborhood around the edge $E_{23}=\left[f_{2}, f_{3}\right]$ (top right) and one around the edge $E_{34}=\left[f_{3}, f_{4}\right]$ (bottom).

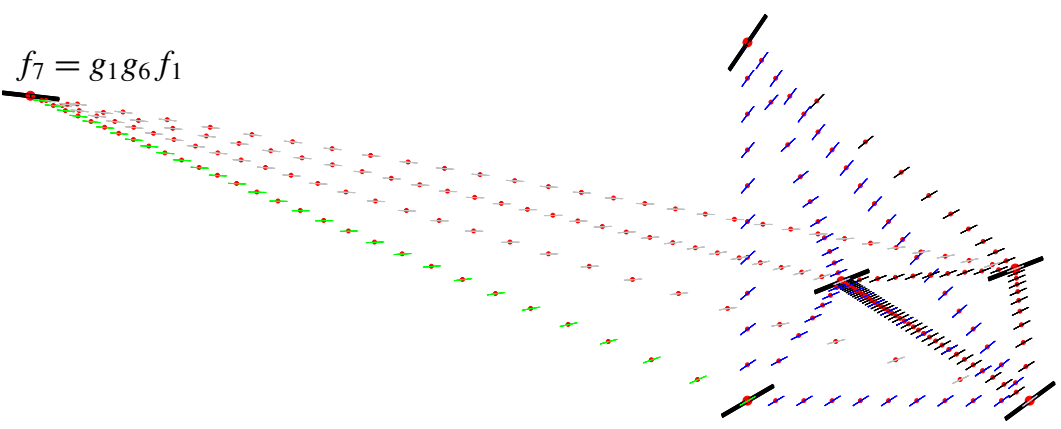

Figure 15. Tetrahedra around the edge $E_{24}$. 


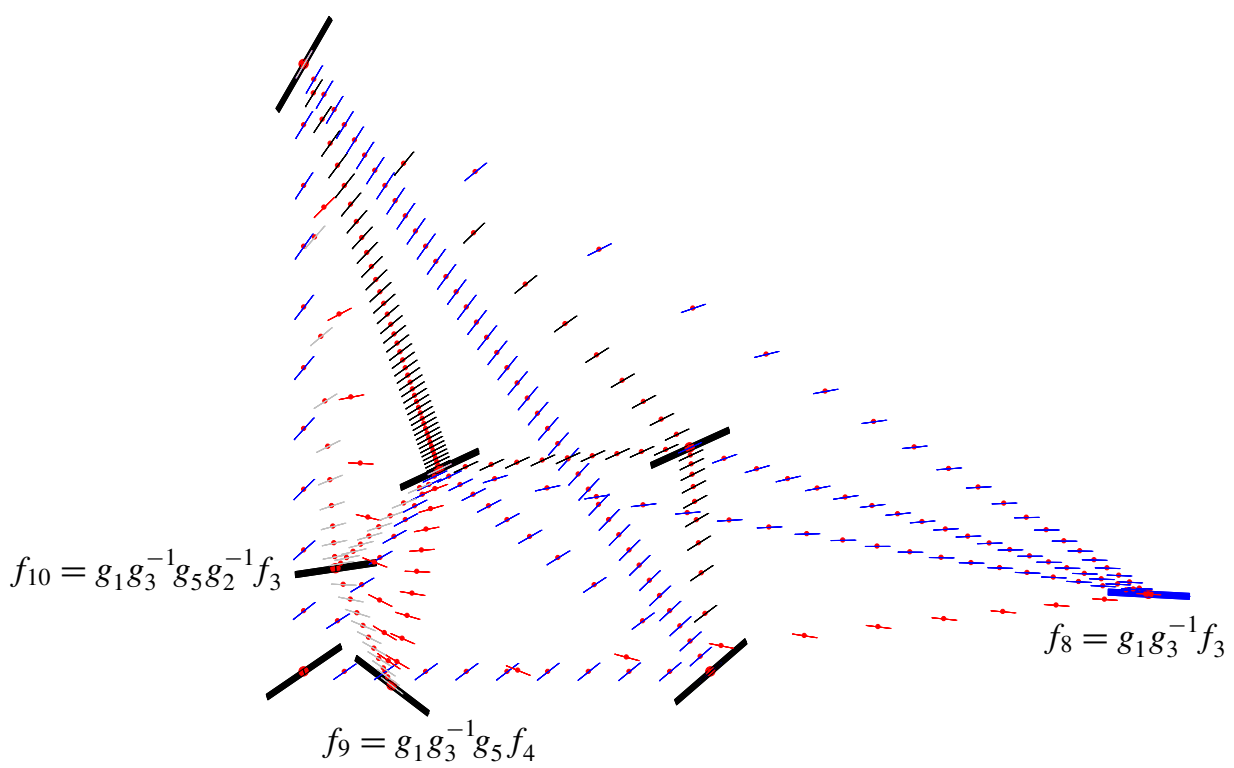

Figure 16. Tetrahedra around the edge $E_{34}$.

$$
\begin{aligned}
& f_{14}=g_{1} g_{2} g_{4}^{-1} g_{5} g_{6}^{-1} \frac{g_{3}^{-1} f_{3}}{f_{12}=g_{1} g_{2} g_{4}^{-1} g_{5} f_{4}} \\
& f_{13}=g_{1} g_{2} g_{4}^{-1} g_{5} g_{6}^{-1} f_{4}
\end{aligned}
$$

Figure 17. Vertices of tetrahedra around the edge $E_{23}$. The group of 6 points in the center can be zoomed to coincide with Figure 13.

As a consequence of the propositions, we obtain our conclusion:

Theorem 6.8. The manifold m009 has a flag structure whose holonomy map is boundary unipotent.

\section{Appendix}

A1. Generators and side pairings. To help the reader check computations we list explicitly the side pairings we use. Note that we simplify notation denoting matrices by the same letters as the maps. First we let

$$
\begin{array}{ll}
u_{1}=1-\frac{1}{u_{12}}=\frac{2}{\gamma+3}, & w_{1}=1-\frac{1}{w_{12}}=\frac{2}{2-\gamma},
\end{array}
$$




$$
\begin{aligned}
& u_{3}=u_{34}=\frac{\gamma-2}{\gamma} \text {, } \\
& w_{3}=w_{34}=\frac{\gamma+3}{\gamma+1}, \\
& v_{3}=v_{34}=\frac{1}{\gamma+3}, \\
& u_{4}=\frac{1}{1-u_{43}}=\frac{1}{2+\gamma}, \\
& w_{4}=\frac{1}{1-w_{43}}=\frac{1}{1-\gamma} \text {, } \\
& v_{4}=\frac{1}{1-v_{43}}=\frac{\gamma-2}{\gamma-1} \text {. }
\end{aligned}
$$

The generators are given by

$$
\begin{aligned}
& F_{234}^{0}=g_{1}\left(F_{243}^{1}\right) \quad g_{1}=\left[\begin{array}{ccc}
-\lambda_{3} & 0 & \lambda_{3} \\
-\lambda_{1}-\lambda_{3} & \lambda_{1} & \lambda_{3} \\
-\lambda_{3}+\lambda_{2} & 0 & \lambda_{3}
\end{array}\right], \quad \begin{array}{l}
\lambda_{2}=\lambda_{1}\left(v_{3}-1\right)\left(1-u_{4}\right), \\
\lambda_{3}=\frac{\lambda_{1}}{\left(v_{4}-1\right)\left(1-u_{3}\right)},
\end{array} \\
& F_{142}^{1}=g_{2}\left(F_{241}^{2}\right), \quad g_{2}=\left[\begin{array}{ccc}
0 & \delta_{3} & \delta_{2}-\delta_{3} \\
\delta_{1} & 0 & \delta_{2}-\delta_{1} \\
0 & 0 & \delta_{2}
\end{array}\right], \quad \begin{array}{c}
\delta_{2}=\frac{\delta_{1} v_{1}\left(w_{2}-1\right)}{w_{2}\left(v_{1}-1\right)}, \\
\delta_{3}=\frac{\delta_{1}\left(1-v_{4}\right)\left(1-w_{4}\right)}{v_{4} w_{4}},
\end{array} \\
& F_{142}^{0}=g_{3}\left(F_{314}^{1}\right), \quad g_{3}=\left[\begin{array}{ccc}
\alpha_{2} & -\alpha_{2}-\alpha_{1} & \alpha_{1} \\
\alpha_{2} & \alpha_{3}-\alpha_{2} & 0 \\
\alpha_{2} & -\alpha_{2} & 0
\end{array}\right], \quad \begin{array}{c}
\alpha_{2}=\frac{\alpha_{1} u_{2} v_{4}}{1-u_{2}}, \\
\alpha_{3}=\frac{\alpha_{1} u_{4}\left(v_{1}-1\right)}{u_{4}-1},
\end{array} \\
& F_{134}^{0}=g_{4}\left(F_{143}^{2}\right), \quad g_{4}=\left[\begin{array}{ccc}
\beta_{1}-\beta_{1}-\beta_{3} & \beta_{3} \\
0 & \beta_{3} & \beta_{3} \\
0 & \beta_{2}-\beta_{3} & \beta_{3}
\end{array}\right], \\
& {\left[\begin{array}{ccc}
0 & \beta_{2}-\beta_{3} & \beta_{3}
\end{array}\right], \quad \beta_{3}=\frac{\beta_{1} u_{3}}{w_{4}\left(1-u_{3}\right)},} \\
& F_{123}^{0}=g_{5}\left(F_{213}^{2}\right), \quad g_{5}=\left[\begin{array}{ccc}
0 & \epsilon_{1} & 0 \\
\epsilon_{2} & 0 & 0 \\
0 & 0 & \epsilon_{3}
\end{array}\right], \quad \begin{array}{c}
\epsilon_{2}=\epsilon_{1} u_{3} w_{3} \\
\epsilon_{3}=\frac{\epsilon_{1} u_{2}}{w_{1}}
\end{array} \\
& F_{123}^{1}=g_{6}\left(F_{342}^{2}\right), \quad g_{6}=\left[\begin{array}{rrr}
-\zeta_{1} & 0 & \zeta_{1} \\
\zeta_{2} & 0 & 0 \\
-\zeta_{3} & \zeta_{3} & 0
\end{array}\right], \quad \begin{array}{l}
\zeta_{2}=\zeta_{1} v_{3}\left(w_{2}-1\right), \\
\zeta_{3}=\zeta_{1} v_{2}\left(1-w_{4}\right),
\end{array}
\end{aligned}
$$

Thinking of the generators as hyperbolic transformations, we can obtain a presentation of the fundamental group of m009. Indeed, the side pairings of the (hyperbolic) polyhedron formed by gluing the tetrahedra (as in Figure 13) according to $g_{1}:(243)^{1} \rightarrow(234)^{0}$ and $g_{2}:(142)^{2} \rightarrow(241)^{1}$ are

$$
s_{3}=g_{3} g_{1}^{-1}, \quad s_{4}=g_{4} g_{2}^{-1} g_{1}^{-1}, \quad s_{5}=g_{5} g_{2}^{-1} g_{1}^{-1}, \quad s_{6}=g_{1} g_{6} g_{2}^{-1} g_{1}^{-1} .
$$

The three edge cycles give the relations

$$
s_{6} s_{3}^{-1}, \quad s_{4}^{-1} s_{5} s_{6}^{-1} s_{3}^{-1} s_{4} s_{5}^{-1}, \quad s_{3}^{-1} s_{5} s_{6} s_{4}^{-1},
$$


and the presentation of the fundamental group $\Gamma=\pi_{1}(\mathrm{~m} 009)$ of the manifold m009 can be simplified to be

$$
\Gamma=\left\langle s_{3}, s_{5} \mid\left[s_{3}^{-1}, s_{5}^{-1}\right] s_{3}^{-2}\left[s_{3}^{-1}, s_{5}\right]\right\rangle .
$$

The manifold m009 is fibered over the circle. From the presentation, we know that its fundamental group $\Gamma$ has abelianization

$$
\Gamma /[\Gamma, \Gamma]=\mathbb{Z} / 2 \mathbb{Z} \oplus \mathbb{Z} .
$$

Indeed, from the presentation we observe that $s_{3}^{2} \in[\Gamma, \Gamma]$. We conclude that the image of $s_{5}$ in $\Gamma /[\Gamma, \Gamma]$ is nontrivial and generates an infinite cyclic group.

One can also check (using SnapPea for instance and comparing fundamental groups) that $\mathrm{m} 009$ is the same as the manifold $\mathrm{b}_{++} \mathrm{RRL}$ which is the punctured torus bundle defined by the pseudo-Anosov

$$
\left[\begin{array}{ll}
3 & 2 \\
1 & 1
\end{array}\right]
$$

However, a computation with the matrices of $s_{3}$ and $s_{5}$ (we warn the reader that we also write $s_{i}$ for the image of $s_{i}$ under the holonomy representation, by abuse of notation) shows that the holonomy group is contained in a triangle group of type $(3,3,5)$. Indeed, $s_{3}$ is of order $5, s_{3} s_{5}$ and $s_{3}^{2} s_{5}$ are of order 3 . On the other hand $s_{5}$ is unipotent.

A2. Slicing tetrahedra and proof of Proposition 6.7. In this section we describe a method to slice a tetrahedron according to directions of the flags contained on it. This is the main technical tool that we used to show the compatibility of the structure around an edge (Proposition 6.7) and can be implemented on a computer to check other examples.

In order to analyze gluings of tetrahedra properly, it will be convenient, in a fixed preferred chart, to deal with "constant angle" flags in a given face:

Definition A3. The constant angle $\theta$ path $C_{123}(\theta)$ in a face $F_{123}$ is the set of flags $f=(p, l) \in F_{123}$ such that, in the preferred chart, all lines $l$ make an angle $\theta$ - see (2.2.1). That is,

$$
C_{123}(\theta)=\left\{f \in F_{123} \mid f=(p, l)=(p,[a, b, c]) \text { with } \tan \theta=(b-a) /(a-c)\right\} .
$$

The following lemma ensures that the set $C_{123}(\theta)$ is a path that, in the extreme cases, has only one flag.

Lemma A4. Let $F_{123}$ be a face with vertices $f_{i}=\left(p_{i}, l_{i}\right), i=1,2,3$ such that, in the preferred chart, each line $l_{i}$ makes an angle $\theta_{i}$ with $\theta_{i} \neq \theta_{j}, i \neq j$. Then, to any angle $\theta$ such that

$$
\min \left\{\theta_{1}, \theta_{2}, \theta_{3}\right\}=\theta_{m} \leq \theta \leq \theta_{M}=\max \left\{\theta_{1}, \theta_{2}, \theta_{3}\right\},
$$


the set $C_{123}(\theta)$ is a curve in the face and so we have the disjoint union

$$
F_{123}=\bigcup_{\theta_{m} \leq \theta \leq \theta_{M}} C_{123}(\theta) .
$$

Proof. We use the preferred chart, and, without loss of generality, we consider the face $F_{312}$ as in Figure 7 . So, $p_{1}=(0,0), p_{2}=(1,0), p_{3}=(0,1)$. Let $l_{1}=\left(a_{1} u, b_{1} u\right), l_{2}=\left(a_{2} v+1, b_{2} v\right)$, and $l_{3}=\left(a_{3} w, b_{3} w+1\right)$ for $u, v, w \in \mathbb{R}$. We first parametrize a flag $(p, l) \in F_{312}$ in terms of the coordinates in the preferred chart. The parametrization is in terms of the coordinates of the point $p$, which, by abuse of notation, we consider already in the preferred coordinate chart. For $p=(y, z) \in \pi_{1}\left(F_{312}\right)$,

$$
\begin{aligned}
\overrightarrow{p_{1} p_{2}} & =\left(r p_{1}+(1-r) p_{2}\right)=((1-r), 0) \\
\overrightarrow{p_{3}} & =\left(s p+(1-s) p_{3}\right)=(s y, s z+(1-s)) .
\end{aligned}
$$

Let $p_{t}=\overrightarrow{p_{1} p_{2}} \cap \overrightarrow{p p_{3}}$, so $p_{t}=(y /(1-z), 0)$. Then,

$$
\begin{aligned}
p_{12} & =l_{1} \cap l_{2}=\left(\frac{a_{1} b_{2}}{a_{1} b_{2}-a_{2} b_{1}}, \frac{b_{1} b_{2}}{a_{1} b_{2}-a_{2} b_{1}}\right), \\
\overrightarrow{p_{t} p_{12}} & =\left(r p_{t}+(1-r) p_{12}\right) \\
& =\left(\frac{r y}{1-z}+\frac{(1-r) a_{1} b_{2}}{a_{1} b_{2}-a_{2} b_{1}}, \frac{(1-r) b_{1} b_{2}}{a_{1} b_{2}-a_{2} b_{1}}\right) .
\end{aligned}
$$

Let $q=\overrightarrow{p_{t} p_{12}} \cap l_{3}$. Then,

$$
\begin{aligned}
& q=\left(q_{y}, q_{z}\right)=\left(\frac{a_{3}\left(y\left(b_{1} b_{2}+a_{2} b_{1}-a_{1} b_{2}\right)+a_{1} b_{2}(1-z)\right)}{b_{3} y\left(a_{1} b_{2}-a_{2} b_{1}\right)+b_{2}(1-z)\left(a_{3} b_{1}-a_{1} b_{3}\right)},\right. \\
&\left.\frac{b_{1} b_{2}\left(y b_{3}+a_{3}(1-z)\right)}{b_{3} y\left(a_{1} b_{2}-a_{2} b_{1}\right)+b_{2}(1-z)\left(a_{3} b_{1}-a_{1} b_{3}\right)}\right) .
\end{aligned}
$$

Since $l=\overrightarrow{p q}$,

$$
\begin{aligned}
\tan \theta & =\frac{q_{z}-z}{q_{y}-y} \\
& =\frac{y b_{3}\left(b_{1} b_{2}-z\left(a_{1} b_{2}-a_{2} b_{1}\right)\right)+a_{3} b_{1} b_{2}(1-z)^{2}+z b_{2} a_{1} b_{3}(1-z)}{y\left(a_{3} b_{1} b_{2}+\left(a_{3}+y b_{3}\right)\left(a_{2} b_{1}-a_{1} b_{2}\right)\right)+b_{2}(1-z)\left(a_{3} a_{1}+y\left(a_{1} b_{3}-a_{3} b_{1}\right)\right)} .
\end{aligned}
$$

Fixing $\theta$, we obtain the equation of a conic in the preferred chart which always contains $p_{3}=(0,1)$. The part of this conic inside $\pi_{1}\left(F_{312}\right)$ is the projection $\pi_{1}\left(C_{312}(\theta)\right)$. As for all $\theta$ such that $\theta_{m} \leq \theta \leq \theta_{M}$, we have $C_{312}(\theta) \neq \varnothing$ and conclude that $F_{312}$ is the disjoint union of paths, as claimed. Notice that $F_{312}$ has only one flag with $\theta=\theta_{m}$ and another one with $\theta=\theta_{M}$. 


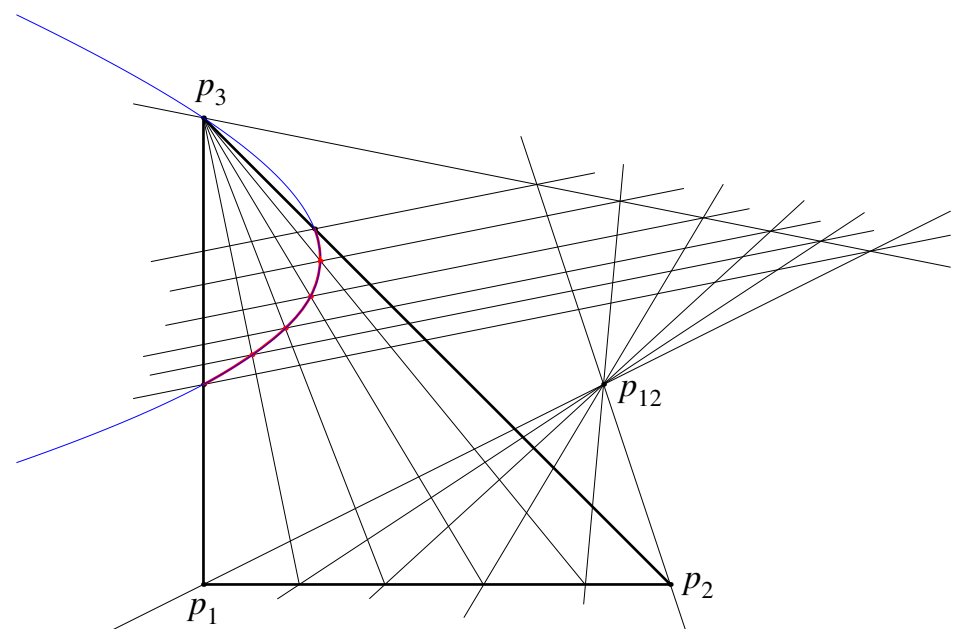

Figure 18. Path $C_{312}(\theta)$ with constant $\theta$ in the face $F_{312}$.

For example, in Figure 18, the red path is $\pi_{1}\left(C_{312}(\theta)\right)$ for $a_{1}=2, b_{1}=1, a_{2}=-1$, $b_{2}=3, a_{3}=5, b_{3}=-1$, and $\tan \theta=1 / 5$. The blue path is a branch of a hyperbola determined by the equation of Lemma A4. Notice that both lines coincide inside $\pi_{1}\left(F_{312}\right)$.

Definition A5. A slice $S_{1234}(\theta)$ with constant $\theta$ in a tetrahedron $T_{1234}$ is the set of flags $f=(p, l) \in T_{1234}$ such that, in the preferred chart, all lines $l$ make an angle $\theta$; that is,

$S_{1234}(\theta)=\left\{f \in T_{1234} \mid f=(p, l)=(p,[a, b, c])\right.$ with $\left.\tan \theta=(b-a) /(a-c)\right\}$.

Proposition A6. Suppose that $T_{1234}$ is a finite tetrahedron with vertices $f_{i}=\left(p_{i}, l_{i}\right)$, $i=1,2,3,4$, such that, in the preferred chart, each line $l_{i}$ makes an angle $\theta_{i}$ with $\theta_{i} \neq \theta_{j}, i \neq j$. Then, for any angle $\theta$ such that

$$
\min \left\{\theta_{1}, \theta_{2}, \theta_{3}, \theta_{4}\right\}=\theta_{m} \leq \theta \leq \theta_{M}=\max \left\{\theta_{1}, \theta_{2}, \theta_{3}, \theta_{4}\right\},
$$

the set $S_{1234}(\theta)$ has three or four vertices (each one in a distinct edge). Furthermore, the tetrahedron is a disjoint union of slices, that is,

$$
T_{1234}=\bigcup_{\theta_{m} \leq \theta \leq \theta_{M}} S_{1234}(\theta) .
$$

Proof. Without loss of generality, we can consider that the tetrahedron $T_{1234}$ is $T_{0}$, as defined in 6.1, represented in the preferred chart in Figure 11 and analyzed in Proposition 6.2. Then, $\theta_{i}$ is such that $\theta_{m}=\theta_{4}<\theta_{1}<\theta_{2}<\theta_{3}=\theta_{M}$, and the tetrahedron's faces are the good faces $F_{314}, F_{342}, F_{412}$, and $F_{312}$. The slices of $T_{0}$ will be denoted $S_{0}(\theta)$. 

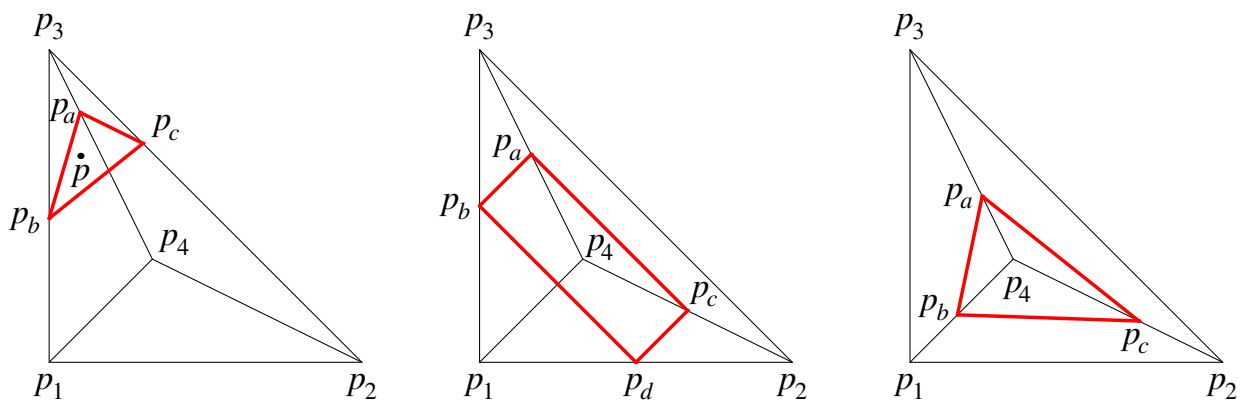

Figure 19. Slices in $T_{0}$ for $\theta_{2} \leq \theta \leq \theta_{3}, \theta_{1} \leq \theta \leq \theta_{2}$, and $\theta_{4} \leq \theta \leq \theta_{1}$.

In the first case, let $\theta$ satisfy $\theta_{2} \leq \theta \leq \theta_{3}=\theta_{M}$. Then, by Lemma A4, there are three curves $C_{314}(\theta), C_{342}(\theta)$, and $C_{312}(\theta)$ in the respective faces with the common vertex $f_{3}$. Considering the common edges between faces, let $f_{a}=C_{314} \cap C_{342} \in E_{34}$, $f_{b}=C_{314} \cap C_{312} \in E_{13}$, and $f_{c}=C_{342} \cap C_{312} \in E_{23}$. We obtain the slices as in Figure 19 (for simplicity the paths are depicted as straight lines).

By the monotonicity lemma (Lemma 3.1), each one of these curves separates the respective faces into two parts: one near the vertex $f_{3}$ where $\theta^{\prime}>\theta$ and the other near the two other vertices of the face where $\theta^{\prime \prime}<\theta$. Consider a point $p$ in $\mathbb{P}\left(\mathbb{R}^{3}\right)$ as in Figure 19. There exist two flags in the faces of $T_{0}$ which project onto $p$, namely, $(p, d) \in F_{312}$ and $\left(p, d^{\prime}\right) \in F_{314}$ with $\theta_{d}>\theta$ and $\theta_{d^{\prime}}<\theta$. Then, by the definition of $T_{0}$ as a 3-simplex (see Proposition 6.2), for all $p$ inside the area delimited by $\pi_{1}\left(C_{312}(\theta)\right), \pi_{1}\left(C_{314}(\theta)\right)$, and $\pi_{1}\left(C_{342}(\theta)\right)$, there exists a flag $f^{\prime}=\left(p, l^{\prime}\right) \in T_{0}$ such that $\theta^{\prime}=\theta$, so $f^{\prime} \in S_{0}(\theta)$. Clearly, $C_{312}(\theta) \subset S_{0}(\theta), C_{314}(\theta) \subset S_{0}(\theta)$, and $C_{342}(\theta) \subset S_{0}(\theta)$. Furthermore $S_{0}(\theta)$ has three vertices: $f_{a} \in E_{34}, f_{b} \in E_{13}$, and $f_{c} \in E_{24}$, as claimed.

In the other cases, as in Figure 19, the argument is the same, and this concludes the first part of the proof.

The second part follows clearly, as for all $\theta$ such that $\theta_{m} \leq \theta \leq \theta_{M}$, we have $S_{0}(\theta) \neq \varnothing$.

Figure 20 shows the exact slices in $T_{0}$ for $\tan \theta=0.4$ and $\tan \theta=0.5$.

Proposition A7. The gluing of the three tetrahedra $T_{0}, g_{1}\left(T_{1}\right)$, and $g_{1} g_{2}\left(T_{2}\right)$ forms a polyhedron in the flag space.

Proof. Observe that $\theta_{5} \leq \theta_{i} \leq \theta_{3}$ for all $i=1, \ldots, 6$. We can construct the slices in each tetrahedron for $\theta$ from $\theta_{5}$ to $\theta_{3}$ and see that they only intersect in the common path contained in the common face between two tetrahedra. In Figure 21, we show the slices for $\tan \theta=0.4\left(\theta_{4}<\theta<\theta_{1}\right), \tan \theta=0.5\left(\theta_{6}<\theta<\theta_{2}\right)$, and $\tan \theta=0.8$ $\left(\theta_{2}<\theta<\theta_{3}\right)$. The other two cases $\theta_{5}<\theta<\theta_{4}$ and $\theta_{1}<\theta<\theta_{6}$ are similar.

Now consider the tetrahedron 2457 with vertices $f_{2}, f_{4}, f_{5}=g_{1}\left[p_{1}, l_{1}\right]$, and $f_{7}=g_{1} g_{6}\left[p_{1}, l_{1}\right]$. It is one of the four tetrahedra around the edge $E_{24}$ in Figure 15. 

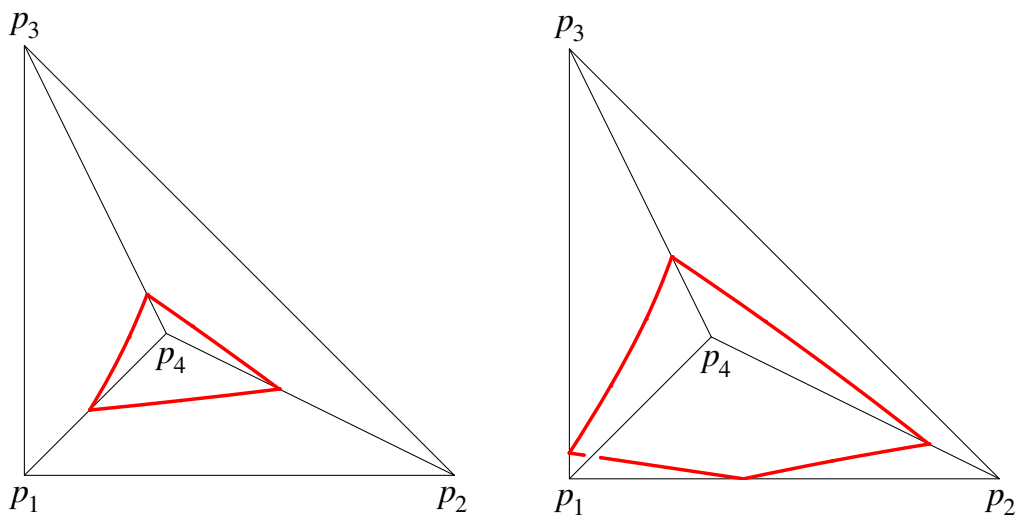

Figure 20. Left: slice in $T_{0}$ for $\tan \theta=0.4$, exemplifying the case $\theta_{4}<\theta<\theta_{1}<\theta_{2}<\theta_{3}$. Right: slice for $\tan \theta=0.5$, corresponding to $\theta_{4}<\theta_{1}<\theta<\theta_{2}<\theta_{3}$.
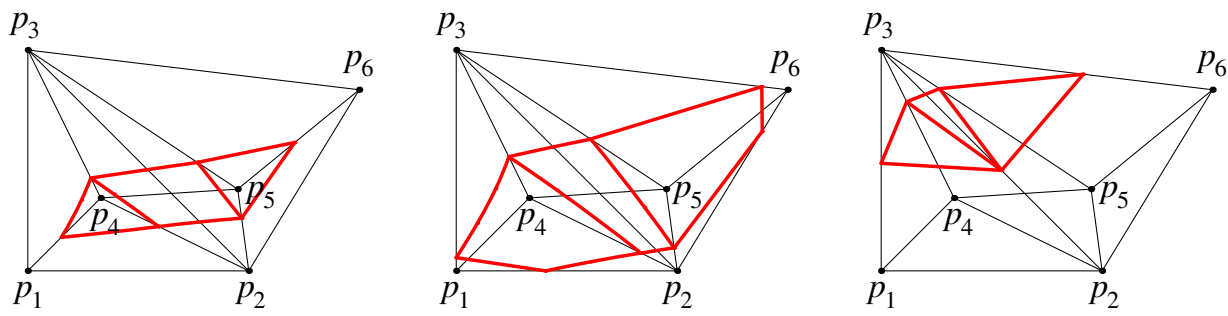

Figure 21. Slices in the gluing of $T_{0}, g_{1}\left(T_{1}\right)$ and $g_{1} g_{2}\left(T_{2}\right)$ for $\tan \theta=0.4$, that is, $\theta_{4}<\theta<\theta_{1}, \tan \theta=0.5$, that is, $\theta_{6}<\theta<\theta_{2}$, and $\tan \theta=0.8$, that is, $\theta_{2}<\theta<\theta_{3}$.

The following lemma describes the position of the projection of certain slices. It will be important in order to construct slices around an edge in the next proposition.

Lemma A8. Consider the tetrahedron 2457, in the preferred chart, and the projection of the path $C_{725}\left(\theta_{p}\right)$, that is $\pi_{1}\left(C_{725}\left(\theta_{p}\right)\right)$. Then, for all $f_{p}=\left(p, l_{p}\right) \in E_{24}$, we have $q=\pi_{1}\left(E_{24}\right) \cap \pi_{1}\left(C_{725}\left(\theta_{p}\right)\right) \in\left[p_{2}, p\right]$.

In other words the projection of the path $C_{725}\left(\theta_{p}\right)$ intersects the projection of the edge $E_{24}$, that is $\pi_{1}\left(E_{24}\right)$, between $p_{2}=\pi_{1}\left(f_{2}\right)$ and $p=\pi_{1}\left(f_{p}\right)$, where $f_{p}=\left(p, l_{p}\right)$ is the flag in the edge $E_{24}$ such that the line $l_{p}$ makes an angle $\theta_{p}$.

Proof. The projection of the tetrahedron is represented in Figure 22 with a detail in the lower diagram. Let $f_{p}=\left(p, l_{p}\right) \in E_{24}$, and let $\theta_{p}$ be the angle of $l_{p}$ in the preferred chart. By definition, $p \in l_{p}$ and $p_{24}=l_{2} \cap l_{4} \in l_{p}$. As $\theta_{7}<\theta_{5}<\theta_{4}<\theta_{p}<\theta_{2}$, the path $C_{725}\left(\theta_{p}\right)$ has one end in $E_{25}$ and another in $E_{27}$, so clearly $\pi_{1}\left(C_{725}\left(\theta_{p}\right)\right)$ intersects $\pi_{1}\left(E_{24}\right)$ or $\pi_{1}\left(E_{45}\right)$. 


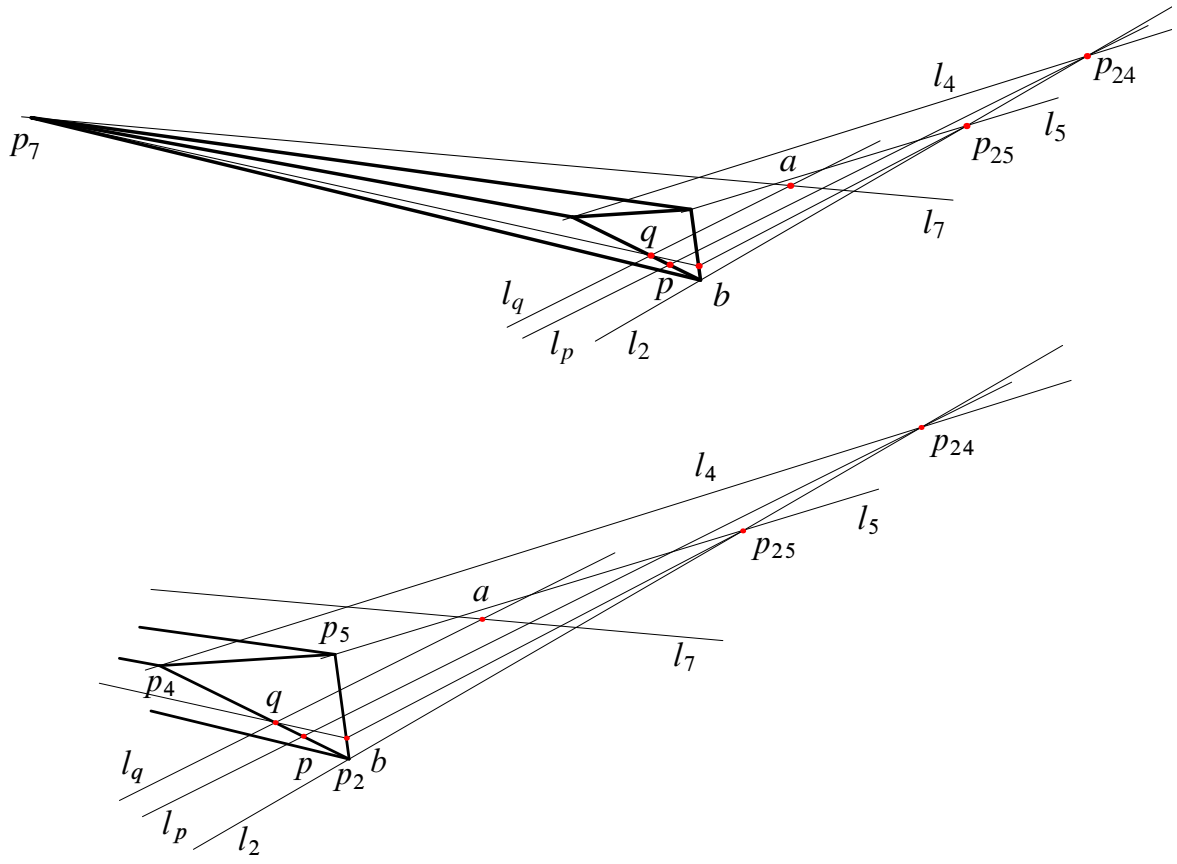

Figure 22. Top: tetrahedron 2457. Relative position of $p$ and $q$ in the edge $E_{24}$. Bottom: detail of the same tetrahedron, showing the relative position of $p$ and $q$ in the edge $E_{24}$.

Suppose first that it intersects $E_{24}$. Let $f_{q}=\left(q, l_{q}\right) \in C_{725}\left(\theta_{p}\right)$ such that $q=$ $\pi_{1}\left(E_{24}\right) \cap \pi_{1}\left(C_{725}\left(\theta_{p}\right)\right)$. Suppose, towards a contradiction, that $q \in\left[p, p_{4}\right]$. By definition, $l_{p}$ and $l_{q}$ are parallel. Let $a=l_{q} \cap l_{7}$ and let $b$ be the intersection between $\pi_{1}\left(E_{25}\right)$ and the straight line through $p_{7}$ and $q$. Notice that $b$ and $p_{25}$ are on opposite sides with respect to $l_{7}$ but are on the same side with respect to $l_{q}$. As $a=l_{q} \cap l_{7}$, the points $a, b$, and $p_{25}$ don't lie in the same straight line, which contradicts the definition of the face $F_{725}$. Indeed, if $f_{q} \in F_{725}$, then $a, b$, and $p_{25}$ should lie in the same straight line. We conclude that $q \notin\left[p, p_{4}\right]$.

By the same construction and arguments we obtain that $\pi_{1}\left(C_{725}\left(\theta_{p}\right)\right)$ doesn't intersect $\pi_{1}\left(E_{45}\right)$. Thus, $\pi_{1}\left(C_{725}\left(\theta_{p}\right)\right)$ intersects $\pi_{1}\left(E_{24}\right)$ between $p$ and $p_{2}$.

Proposition A9. Along the edge $E_{24}$, the gluing of the tetrahedra defines a neighborhood.

Proof. Consider the gluing of four tetrahedra along $E_{24}$, represented in Figure 15. Consider also the tetrahedron $g_{1} g_{6}\left(T_{2}\right)$ with vertices $f_{2}, f_{4}, f_{5}$, and $f_{7}$ such that $\theta_{7}<\theta_{5}<\theta_{4}<\theta_{1}<\theta_{2}$. Observe first that, by Lemma A8, for all $\theta$ such that $\theta_{4}<$ $\theta<\theta_{2}$ (that is, $\tan \theta_{4} \approx 0.313<\tan \theta<\tan \theta_{2} \approx 0.580$ ), the slice $S_{2457}(\theta)$ projects 


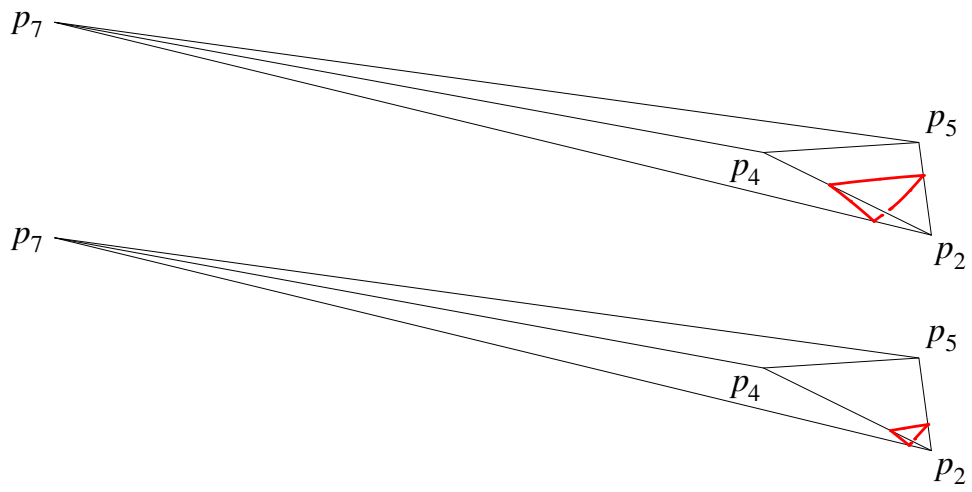

Figure 23. Slice $S_{2457}(\theta)$ in $g_{1} g_{6}\left(T_{2}\right)$ for $\tan \theta=0.4$ (top) and $\tan \theta=0.5$ (bottom).

to a preferred chart in a "triangle" with vertices in the edges $\left[p_{7}, p_{2}\right],\left[p_{2}, p_{4}\right]$, and $\left[p_{2}, p_{5}\right]$, as shown in Figure 23 for $\tan \theta=0.4<\tan \theta_{1} \approx 0.458$ (top diagram) and $\tan \theta=0.5>\tan \theta_{1}$ (bottom).

As a consequence, the unique form of the slices $S_{2457}(\theta)$ (they all project to the triangles described above), the form of the slices $S_{1234}(\theta), S_{1247}(\theta)$, and $S_{2345}(\theta)$ are unique too. Indeed, the slices have common paths, for example, $C_{425}(\theta)=$ $S_{2457}(\theta) \cap S_{2345}(\theta)$, and two paths inside a tetrahedron don't intersect. Therefore, we obtain slices in each one of the four tetrahedra as in Figure 24 for $\tan \theta=0.4<\tan \theta_{1}$ and in Figure 25 for $\tan \theta=0.5>\tan \theta_{1}$. Thus, all neighborhoods of a point $p \in \pi_{1}\left(E_{24}\right)$ are as in Figure 26 for $\theta_{4}<\theta<\theta_{1}<\theta_{2}$ or as in Figure 27 for $\theta_{4}<\theta_{1}<\theta<\theta_{2}$. Figures 23 to 27 are obtained through exact computations.
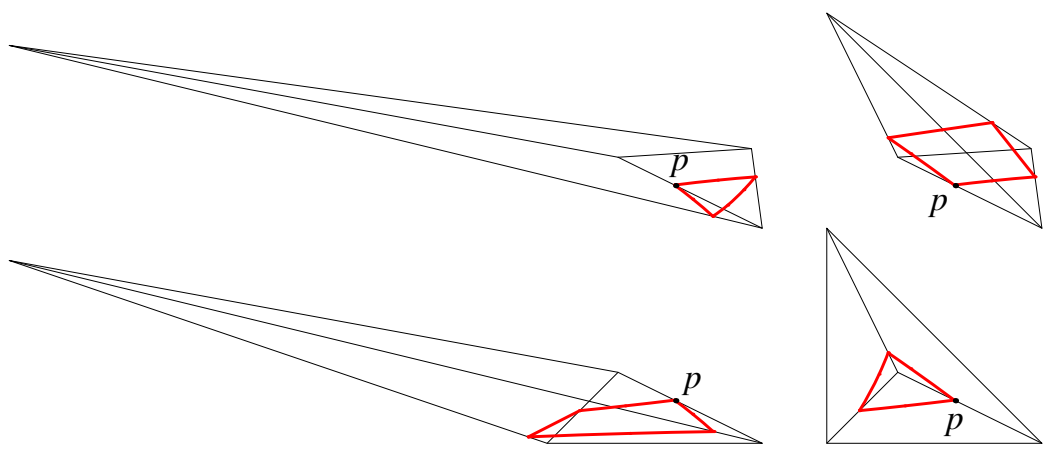

Figure 24. Slices in each tetrahedron with common edge $E_{24}$ for $\tan \theta=0.4$. 

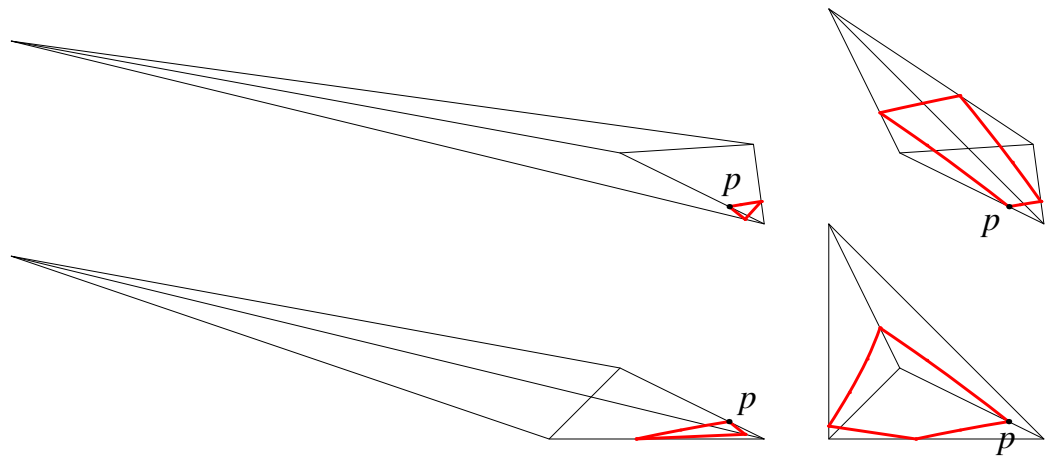

Figure 25. Slices in each tetrahedron with common edge $E_{24}$ for $\tan \theta=0.5$.

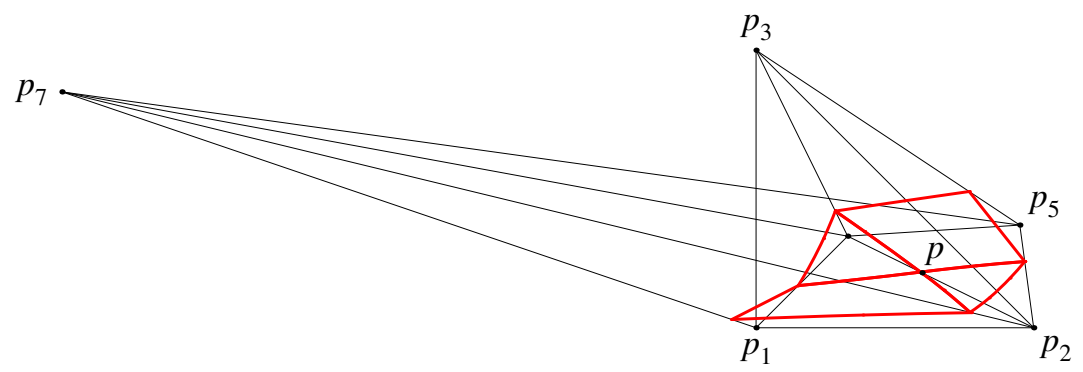

Figure 26. Neighborhood of $p$ such that $f=(p, l) \in E_{24}$ for $\theta_{7}<\theta_{5}<\theta_{4}<\theta<\theta_{1}<\theta_{2}<\theta_{3}$.

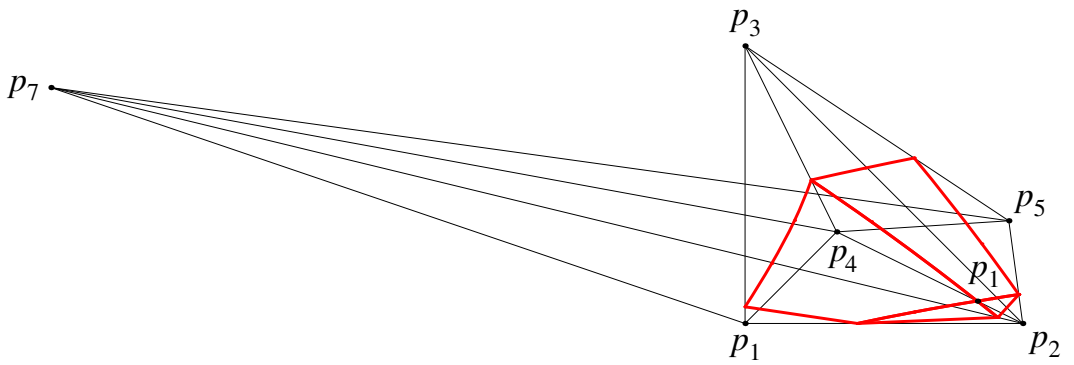

Figure 27. Neighborhood of $p$ such that $f=(p, l) \in E_{24}$ for $\theta_{7}<\theta_{5}<\theta_{4}<\theta_{1}<\theta<\theta_{2}<\theta_{3}$.

\section{Acknowledgments}

We thank N. Bergeron, M. Deraux, A. Guilloux, Neil Hoffman, and P.-V. Koseleff for stimulating discussions. We also thank an anonymous referee for many suggestions that improved the exposition. 
This work was partially financed by an ANR project SGT (Structures géométriques triangulées). Santos Thebaldi is supported by CAPES Brazil (BEX 9599/11-8) and partially supported by UFOP. He also thanks FAPEMIG Brazil for financial support (CEX-APQ-01056-08).

\section{References}

[Barbot 2001] T. Barbot, "Flag structures on Seifert manifolds", Geom. Topol. 5 (2001), 227-266. MR 2002h:57035 Zbl 1032.57037

[Bergeron et al. 2014] N. Bergeron, E. Falbel, and A. Guilloux, "Tetrahedra of flags, volume and homology of SL(3)”, Geom. Topol. 18:4 (2014), 1911-1971. MR 3268771 Zbl 06356603

[Cooper et al. 2015] D. Cooper, D. D. Long, and S. Tillmann, "On convex projective manifolds and cusps", Adv. Math. 277 (2015), 181-251. MR 3336086 Zbl 06431971

[Deraux 2015] M. Deraux, "On Spherical CR Uniformization of 3-Manifolds”, Exp. Math. 24:3 (2015), 355-370. MR 3359222

[Falbel 2008] E. Falbel, "A spherical CR structure on the complement of the figure eight knot with discrete holonomy", J. Differential Geom. 79:1 (2008), 69-110. MR 2009d:32037 Zbl 1148.57025

[Falbel et al. 2015] E. Falbel, P.-V. Koseleff, and F. Rouillier, "Representations of fundamental groups of 3-manifolds into PGL(3, C): exact computations in low complexity", Geom. Dedicata 177 (2015), 229-255. MR 3370032 Zbl 06468790

[Falbel et al. 2015-] E. Falbel, S. Garoufalidis, A. Guilloux, M. Görner, P.-V. Koseleff, F. Rouillier, and C. Zickert, "CURVE", electronic reference, 2015-, Available at http://curve.unhyperbolic.org.

[Fock and Goncharov 2007] V. V. Fock and A. B. Goncharov, "Moduli spaces of convex projective structures on surfaces", Adv. Math. 208:1 (2007), 249-273. MR 2008g:57015 Zbl 1111.32013

[Garoufalidis et al. 2011] S. Garoufalidis, D. Thurston, and C. Zickert, "The complex volume of $\operatorname{SL}(n, \mathbb{C})$-representations of 3-manifolds", preprint, 2011. arXiv 1111.2828v2

[Gelfand and MacPherson 1982] I. M. Gelfand and R. D. MacPherson, "Geometry in Grassmannians and a generalization of the dilogarithm", Adv. in Math. 44:3 (1982), 279-312. MR 84b:57014

[Thurston 1981] W. Thurston, "The geometry and topology of 3-manifolds", Lecture notes, Princeton, 1981, Available at http://library.msri.org/books/gt3m.

Received April 26, 2014. Revised April 14, 2015.

ELISHA FALBEL

Institut DE MAThÉMATiQUes

Université Pierre et MARIE CURIE

75252 PARIS CEDEX 5

FRANCE

elisha.falbel@imj-prg.fr

\section{RAFAEL SANTOS THEBALDI}

Departamento de CiênCIAs Exatas e Aplicadas

Universidade Federal de OURo Preto

35931-008 JoÃo MonleVAde, MG

BRAZIL

rafaelsantosthe@yahoo.com.br

rafael@decea.ufop.br 


\title{
PACIFIC JOURNAL OF MATHEMATICS
}

\author{
msp.org/pjm
}

Founded in 1951 by E. F. Beckenbach (1906-1982) and F. Wolf (1904-1989)

\section{EDITORS}

Don Blasius (Managing Editor)

Department of Mathematics

University of California

Los Angeles, CA 90095-1555

blasius@math.ucla.edu

\author{
Paul Balmer \\ Department of Mathematics \\ University of California \\ Los Angeles, CA 90095-1555 \\ balmer@math.ucla.edu \\ Robert Finn \\ Department of Mathematics \\ Stanford University \\ Stanford, CA 94305-2125 \\ finn@math.stanford.edu \\ Sorin Popa \\ Department of Mathematics \\ University of California \\ Los Angeles, CA 90095-1555 \\ popa@math.ucla.edu
}

\author{
Vyjayanthi Chari \\ Department of Mathematics \\ University of California \\ Riverside, CA 92521-0135 \\ chari@math.ucr.edu \\ Kefeng Liu \\ Department of Mathematics \\ University of California \\ Los Angeles, CA 90095-1555 \\ liu@math.ucla.edu \\ Jie Qing \\ Department of Mathematics \\ University of California \\ Santa Cruz, CA 95064 \\ qing@ cats.ucsc.edu
}

\section{PRODUCTION}

Silvio Levy, Scientific Editor, production@msp.org

\section{SUPPORTING INSTITUTIONS}

ACADEMIA SINICA, TAIPEI

CALIFORNIA INST. OF TECHNOLOGY

INST. DE MATEMÁTICA PURA E APLICADA

KEIO UNIVERSITY

MATH. SCIENCES RESEARCH INSTITUTE

NEW MEXICO STATE UNIV.

OREGON STATE UNIV.

\author{
STANFORD UNIVERSITY \\ UNIV. OF BRITISH COLUMBIA \\ UNIV. OF CALIFORNIA, BERKELEY \\ UNIV. OF CALIFORNIA, DAVIS \\ UNIV. OF CALIFORNIA, LOS ANGELES \\ UNIV. OF CALIFORNIA, RIVERSIDE \\ UNIV. OF CALIFORNIA, SAN DIEGO \\ UNIV. OF CALIF., SANTA BARBARA
}

\author{
Daryl Cooper \\ Department of Mathematics \\ University of California \\ Santa Barbara, CA 93106-3080 \\ cooper@math.ucsb.edu \\ Jiang-Hua Lu \\ Department of Mathematics \\ The University of Hong Kong \\ Pokfulam Rd., Hong Kong \\ jhlu@maths.hku.hk \\ Paul Yang \\ Department of Mathematics \\ Princeton University \\ Princeton NJ 08544-1000 \\ yang@math.princeton.edu
}

These supporting institutions contribute to the cost of publication of this Journal, but they are not owners or publishers and have no responsibility for its contents or policies.

See inside back cover or msp.org/pjm for submission instructions.

The subscription price for 2015 is US \$420/year for the electronic version, and \$570/year for print and electronic.

Subscriptions, requests for back issues and changes of subscribers address should be sent to Pacific Journal of Mathematics, P.O. Box 4163, Berkeley, CA 94704-0163, U.S.A. The Pacific Journal of Mathematics is indexed by Mathematical Reviews, Zentralblatt MATH, PASCAL CNRS Index, Referativnyi Zhurnal, Current Mathematical Publications and Web of Knowledge (Science Citation Index).

The Pacific Journal of Mathematics (ISSN 0030-8730) at the University of California, c/o Department of Mathematics, 798 Evans Hall \#3840, Berkeley, CA 94720-3840, is published twelve times a year. Periodical rate postage paid at Berkeley, CA 94704, and additional mailing offices. POSTMASTER: send address changes to Pacific Journal of Mathematics, P.O. Box 4163, Berkeley, CA 94704-0163.

PJM peer review and production are managed by EditFLOW ${ }^{\circledR}$ from Mathematical Sciences Publishers.

\section{PUBLISHED BY}

\section{mathematical sciences publishers \\ nonprofit scientific publishing}

http://msp.org/

(C) 2015 Mathematical Sciences Publishers 


\section{PACIFIC JOURNAL OF MATHEMATICS}

Volume $278 \quad$ No. $1 \quad$ November 2015

Growth tight actions

Goulnara N. Arzhantseva, Christopher H. Cashen and JING TAO

A flag structure on a cusped hyperbolic 3-manifold

ELISHA FALBEL and RAFAEL SANTOS THEBALDI

A new upper bound for the Dirac operators on hypersurfaces

Nicolas GinouX, GeORGES Habib and Simon RaUlot

Games and elementary equivalence of $\mathrm{II}_{1}$-factors

ISAAC GOLDBRING and THOMAS SINCLAIR

Grossberg-Karshon twisted cubes and hesitant walk avoidance

MEGUMI HARADA and EUNJEONG LEE

Gamma factors of distinguished representations of $\mathrm{GL}_{n}(\mathbb{C})$

ALEXANDER KEMARSKY

The $W$-entropy formula for the Witten Laplacian on manifolds with 173 time dependent metrics and potentials

SONGZI LI and XIANG-DONG LI

A diagrammatic categorification of the affine $q$-Schur algebra $\hat{\boldsymbol{S}}(n, n) 201$ for $n \geq 3$

MARCO MACKAAY and ANNE-LAURE THIEL

Showing distinctness of surface links by taking 2-dimensional braids

INASA NAKAMURA

Correction to Modular $L$-values of cubic level 